University of Wollongong

Research Online

Faculty of Engineering and Information

Faculty of Engineering and Information

Sciences - Papers: Part B

Sciences

2017

\title{
Microdosimetry of electrons in liquid water using the low-energy models of Geant4
}

Ioanna Kyriakou

University of loannina

Dimitris Emfietzoglou

University of loannina

Vladimir N. Ivanchenko

Geant4 Associates International Ltd

M Bordage

University of Toulouse I

Susanna Guatelli

University of Wollongong, susanna@uow.edu.au

See next page for additional authors

Follow this and additional works at: https://ro.uow.edu.au/eispapers1

Part of the Engineering Commons, and the Science and Technology Studies Commons

Research Online is the open access institutional repository for the University of Wollongong. For further information contact the UOW Library: research-pubs@uow.edu.au 


\title{
Microdosimetry of electrons in liquid water using the low-energy models of Geant4
}

\author{
Abstract \\ The biological effects of ionizing radiation at the cellular level are frequently studied using the well-known \\ formalism of microdosimetry, which provides a quantitative description of the stochastic aspects of \\ energy deposition in irradiated media. Energy deposition can be simulated using Monte Carlo codes, \\ some adopting a computationally efficient condensed-history approach, while others follow a more \\ detailed track-structure approach. In this work, we present the simulation of microdosimetry spectra and \\ related quantities (frequency-mean and dose-mean lineal energies) for incident monoenergetic electrons \\ (50 eV-10 keV) in spheres of liquid water with dimensions comparable to the size of biological targets: \\ base pairs ( $2 \mathrm{~nm}$ diameter), nucleosomes $(10 \mathrm{~nm})$, chromatin fibres $(30 \mathrm{~nm})$ and chromosomes (300 $\mathrm{nm})$. \\ Simulations are performed using the condensed-history low-energy physics models ("Livermore" and \\ "Penelope") and the track-structure Geant4-DNA physics models, available in the Geant4 Monte Carlo \\ simulation toolkit. The spectra are compared and the influence of simulation parameters and different \\ physics models, with emphasis on recent developments, is discussed, underlining the suitability of \\ Geant4-DNA models for microdosimetry simulations. It is further shown that with an appropriate choice \\ of simulation parameters, condensed-history transport may yield reasonable results for sphere sizes as \\ small as a few tens of a nanometer.

\section{Disciplines} \\ Engineering | Science and Technology Studies

\section{Publication Details} \\ Kyriakou, I., Emfietzoglou, D., Ivanchenko, V., Bordage, M. C., Guatelli, S., Lazarakis, P., Tran, H. N. \& Incerti, \\ S. (2017). Microdosimetry of electrons in liquid water using the low-energy models of Geant4. Journal of \\ Applied Physics, 122 (2), 024303-1-024303-17.
}

\section{Authors}

loanna Kyriakou, Dimitris Emfietzoglou, Vladimir N. Ivanchenko, M Bordage, Susanna Guatelli, Peter Lazarakis, H Tran, and Sebastien Incerti 


\section{Microdosimetry of electrons in liquid water using the low-energy models of Geant4}

I. Kyriakou, D. Emfietzoglou, V. Ivanchenko, M. C. Bordage, S. Guatelli, P. Lazarakis, H. N. Tran, and S. Incerti

Citation: Journal of Applied Physics 122, 024303 (2017); doi: 10.1063/1.4992076

View online: http://dx.doi.org/10.1063/1.4992076

View Table of Contents: http://aip.scitation.org/toc/jap/122/2

Published by the American Institute of Physics

\section{Articles you may be interested in}

Calculation of lineal energies for water and DNA bases using the Rudd model cross sections integrated within the Geant4-DNA processes

Journal of Applied Physics 122, 014701 (2017); 10.1063/1.4990293

Non-equilibrium microscale thermomechanical modeling of bimetallic particulate fractal structures during ball milling fabrication

Journal of Applied Physics 122, 025118 (2017); 10.1063/1.4993174

Stability and electronic structure of defect complexes in Gd-doped GaN: First-principles calculations

Journal of Applied Physics 122, 023901 (2017); 10.1063/1.4993452

Mechanisms of carbon dimer formation in colliding laser-produced carbon plasmas

Journal of Applied Physics 122, 023303 (2017); 10.1063/1.4993188

Role of temperature in the radiation stability of yttria stabilized zirconia under swift heavy ion irradiation: A study from the perspective of nuclear reactor applications

Journal of Applied Physics 122, 025902 (2017); 10.1063/1.4993177

Electron microscopic observations of Rb particles and pitting in ${ }^{129}$ Xe spin-exchange optical pumping cells Journal of Applied Physics 122, 024902 (2017); 10.1063/1.4991642

\section{Scilight}

Sharp, quick summaries illuminating the latest physics research

\section{Sign up for FREE!}

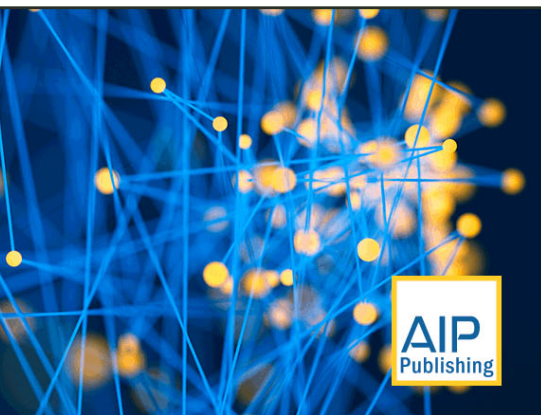




\title{
Microdosimetry of electrons in liquid water using the low-energy models of Geant4
}

\author{
I. Kyriakou, ${ }^{1}$ D. Emfietzoglou, ${ }^{1}$ V. Ivanchenko, ${ }^{2}$ M. C. Bordage,${ }^{3,4}$ S. Guatelli, ${ }^{5}$ P. Lazarakis, ${ }^{5}$ \\ H. N. Tran, ${ }^{6,7}$ and S. Incerti ${ }^{6,7,8,9, a)}$ \\ ${ }^{1}$ Medical Physics Laboratory, University of Ioannina Medical School, 45110 Ioannina, Greece \\ ${ }^{2}$ Geant4 Associates International Ltd., Hebden Bridge, United Kingdom \\ ${ }^{3}$ Université Toulouse III-Paul Sabatier, UMR1037 CRCT, Toulouse, France \\ ${ }^{4}$ Inserm, UMR1037 CRCT, Toulouse, France \\ ${ }^{5}$ Centre for Medical Radiation Physics, University of Wollongong, Wollongong, NSW, Australia \\ ${ }^{6}$ Division of Nuclear Physics, Ton Duc Thang University, Ho Chi Minh City, Vietnam \\ ${ }^{7}$ Faculty of Applied Sciences, Ton Duc Thang University, Ho Chi Minh City, Vietnam \\ ${ }^{8}$ Univ. Bordeaux, CENBG, UMR 5797, F-33170 Gradignan, France \\ ${ }^{9}$ CNRS, IN2P3, CENBG, UMR 5797, F-33170 Gradignan, France
}

(Received 3 March 2017; accepted 24 June 2017; published online 14 July 2017)

\begin{abstract}
The biological effects of ionizing radiation at the cellular level are frequently studied using the well-known formalism of microdosimetry, which provides a quantitative description of the stochastic aspects of energy deposition in irradiated media. Energy deposition can be simulated using Monte Carlo codes, some adopting a computationally efficient condensed-history approach, while others follow a more detailed track-structure approach. In this work, we present the simulation of microdosimetry spectra and related quantities (frequency-mean and dose-mean lineal energies) for incident monoenergetic electrons $(50 \mathrm{eV}-10 \mathrm{keV})$ in spheres of liquid water with dimensions comparable to the size of biological targets: base pairs $(2 \mathrm{~nm}$ diameter), nucleosomes $(10 \mathrm{~nm})$, chromatin fibres $(30 \mathrm{~nm})$ and chromosomes $(300 \mathrm{~nm})$. Simulations are performed using the condensed-history low-energy physics models ("Livermore" and "Penelope") and the track-structure Geant4-DNA physics models, available in the Geant4 Monte Carlo simulation toolkit. The spectra are compared and the influence of simulation parameters and different physics models, with emphasis on recent developments, is discussed, underlining the suitability of Geant4-DNA models for microdosimetry simulations. It is further shown that with an appropriate choice of simulation parameters, condensed-history transport may yield reasonable results for sphere sizes as small as a few tens of a nanometer. Published by AIP Publishing. [http://dx.doi.org/10.1063/1.4992076]
\end{abstract}

\section{INTRODUCTION}

The biological effects of ionizing radiation at the cellular level are often studied using the well-known formalism of microdosimetry, which provides a quantitative description of the stochastic aspects of energy deposition in irradiated media. ${ }^{1}$ These aspects become increasingly more pronounced at low doses and/or targets of small size. Thus, microdosimetry has been traditionally used for radiation quality evaluations in radiation protection, where low doses are of concern, and for mechanistic studies of radiation action at the DNA level. ${ }^{2}$ More recently, the relative biological effectiveness (RBE) problem encountered in radiotherapeutic applications of high-linear energy transfer (LET) radiations (e.g., in hadron therapy) has also been approached within the context of microdosimetry with promising outcomes. ${ }^{3-7}$ Notable examples of microscopic radiobiological models are the Local Effect Model $^{8,9}$ that is used clinically for treatment planning at the Heidelberg Ionbeam Therapy center, Germany and the Microdosimetric Kinetic Model $^{10}$ used at the Heavy Ion Medical Accelerator of NIRS in Chiba, Japan. Both models use as input the

\footnotetext{
a) Author to whom correspondence should be addressed: sebastien.incerti@ tdt.edu.vn and incerti@cenbg.in2p3.fr
}

radiation dose distribution to microscopic volumes and radiobiological data at the subcellular and DNA level.

The Monte Carlo (MC) technique represents a wellestablished theoretical tool in microdosimetry since it enables stochastic simulation of radiation transport (and energy deposition) in the irradiated medium. There exist many MC codes for radiation transport simulation which may be broadly classified as condensed-history $(\mathrm{CH})$ or track-structure (TS) codes. ${ }^{11}$ The distinction is based on the method used for simulating the transport of electrons (primary or secondary) which represent the main computational burden, irrespective of the primary radiation. ${ }^{12}$ In the $\mathrm{CH}$ method, electron transport is based on artificial steps which represent tracksegments that are sufficiently long compared to the electron mean free path. The total effect (energy deposition or angular deflection) of the interactions taking place along each step is estimated by an appropriate multiple-scattering theory such as, for example, the stopping power theory of energy-loss. ${ }^{12}$ This method is particularly effective in reducing the simulation time, thus, rendering electron transport feasible up to very high energies (MeV-GeV). Most of the general-purpose codes, such as MCNP, ${ }^{13} \mathrm{EGS}^{14}{ }^{14}$ GEANT4, ${ }^{15-17}$ FLUKA, $^{18}$ and PENELOPE, ${ }^{19}$ belong to this category. With enough statistics (particle histories), an excellent "spatial resolution" 
can be achieved using $\mathrm{CH}$ techniques; however, they mostly fail to reveal the intrinsic pattern of local energy deposition. Moreover, by construction, multiple-scattering theories are valid for high-energy electrons, practically above about $1 \mathrm{keV} .{ }^{20}$ For improving the spatial resolution, some $\mathrm{CH}$ codes, like EGS, Geant4, and PENELOPE, adopt a "mixed" approach whereby "soft" collisions are still treated by multiple-scattering theories but "hard" collisions are simulated individually by single-scattering cross sections. ${ }^{21,22}$ In principle, setting the hard collision threshold equal to zero should enable mixed $\mathrm{CH}$ codes to simulate all collisions in a discrete manner, thus, resembling a TS code. This is the case of the PENELOPE code where a number of group ${ }^{23,24}$ have investigated its performance in microdosimetry. Despite the fact that PENELOPE employs the most sophisticated inelastic model among $\mathrm{CH}$ codes, the results have revealed that significant artifacts can appear in certain cases. This is an expected outcome, since the cross sections for inelastic scattering (e.g., for $\delta$-ray production) used in mixed $\mathrm{CH}$ codes do not account for the electronic structure of the target in a realistic manner and, therefore, are ill-suited for low-energy electrons. Thus, mixed $\mathrm{CH}$ codes are not considered very reliable for simulating electron transport well-below $1 \mathrm{keV}$, and certainly not below $100 \mathrm{eV} .{ }^{25}$ TS codes, on the other hand, are specific-purpose codes that provide a detailed (event-byevent) description of electron transport down to the $\mathrm{eV}$ range with atomic (or molecular) resolution. ${ }^{26}$ Thus, TS codes are well-suited for microdosimetry down to the DNA level. Among more than a dozen of such codes (reviewed by Nikjoo et al. ${ }^{11}$ ), KURBUC ${ }^{27}$ and PARTRAC ${ }^{28}$ are perhaps the most sophisticated by including elaborate models of critical sub-cellular structures as well as explicit DNA damage and repair pathways. ${ }^{29}$ Results of TS simulations have been imported to the Monte Carlo Damage Simulation software to carry out fast DNA damage calculations. ${ }^{30}$

The main challenge in the development of a TS code is the physics of low-energy electrons since, at sub-keV energies, the interaction cross sections become much more sensitive to the atomic and electronic structure of the material, rendering various high-energy approximations invalid. For example, whereas at high electron energies $(>1 \mathrm{keV})$ the energy-loss rate (or stopping power) is essentially determined by a single-material parameter, the so-called mean excitation energy (or I-value), at low energies the complete oscillator strength distribution of the medium (as a function of both energy- and momentum-transfer) must be known. ${ }^{31}$

The first generation of TS codes employed interaction cross sections based on experimental data for gaseous water which were then scaled linearly to the density of liquid water (typically at $1 \mathrm{~g} \mathrm{~cm}^{-3}$ ). This so-called gas-phase approximation, although valid at high energies, neglects condensedphase effects which are important at low energies and are generally a non-linear function of the material's density. ${ }^{32}$ The gas-phase approximation is, therefore, not suitable at the cellular and DNA level where low-energy electrons are most effective. This realization led to the second generation of TS codes which employ interaction cross sections that are specific to the liquid phase of water, which is used as a surrogate to the cellular medium. ${ }^{33}$ For example, the inelastic cross sections used for electron-impact ionization and electronic excitation in the most known TS codes are calculated from a model dielectric response function of liquid water; specifically, NOREC uses Ritchie's model, ${ }^{34}$ PARTRAC uses Dingfelder's model, ${ }^{35}$ and KURBUC uses Emfietzoglou's model. ${ }^{36}$

In parallel, during recent years, the general-purpose Geant 4 simulation toolkit ${ }^{15-17}$ has been extended with a set of models (the so-called "Geant4-DNA" models, assembled into "physics constructors") allowing an event-by-event description of physical electromagnetic interactions of electrons, protons, neutral hydrogen, alpha particles and their charged states, and a few ions, down to the $\mathrm{eV}$ scale in molecular liquid water medium. ${ }^{37-39}$ While other sets of atomistic models are already available in Geant 4 for the $\mathrm{CH}$ simulation of particle-matter interactions down to a few hundreds of eV (the so-called "Livermore" and "Penelope" physics models), the Geant4-DNA models allow, for the first time, full TS simulations in liquid water. The performance of these TS models and some applications found in the literature have been recently reviewed, ${ }^{37,40}$ but a detailed evaluation of their performance in microdosimetry, especially in comparison to the low energy CH models of Geant4, is still lacking. So far, only a few studies on the usage of Geant4DNA for microdosimetry simulations have been published. Francis $e t \mathrm{al}^{41}$ were the first to present lineal energy distributions and frequency-mean lineal energies for protons in micrometric spheres of liquid water and found a reasonable agreement with experimental data. In a later work, ${ }^{42}$ such distributions were also presented for alpha particles and carbon ions with different linear energy transfer (LET) values. Using a dedicated clustering algorithm and frequency-mean lineal energy values, they could predict larger RBE values for protons compared to alpha particles and carbon ions of the same LET, as previously reported in the literature. More recently, Burigo et $a l .{ }^{43}$ demonstrated that Geant4-DNA models can be used to simulate proton microdosimetry spectra in a microscopic volume of liquid water, in agreement with measured spectra in a wall-less tissue equivalent proportional counter.

In this work, we compare for the first time microdosimetry spectra and related quantities for monoenergetic electrons $(50 \mathrm{eV}-10 \mathrm{keV})$ in small-size spheres of liquid water, with dimensions similar to biological targets, using the Geant 4 simulation toolkit. The performance and limitations of the low-energy electromagnetic physics models (Livermore, Penelope, Geant4-DNA) available in Geant4 as "physics constructors" for the simulation of electron tracks in liquid water are investigated. A dedicated Geant4 example (socalled "microyz" extended example), on which the described simulations are based, will be made available to Geant 4 users after publication of this work.

\section{METHODS}

\section{A. Low-energy electromagnetic models of Geant4}

The Geant 4 toolkit was initially developed for the simulation of high-energy experiments at CERN. ${ }^{44,45}$ Thanks to its object-oriented technology $(\mathrm{C}++$ architecture), the 
application domain of Geant4 progressively and naturally broadened toward other fields requiring particle-matter simulations, such as space science and medical physics. ${ }^{46}$ In particular, the toolkit benefited from the addition of electromagnetic physics models allowing a more accurate description of particlematter interactions in the low energy domain, especially below the $\mathrm{MeV}$ range. These models, which enable mixed $\mathrm{CH}$ simulation, come in two flavors, the "Livermore" and the "Penelope" models and they are available to Geant4 users through two dedicated physics constructors called "G4EmLivermorePhysics" and "G4EmPelenopePhysics," respectively.

\section{1. "Livermore" models}

The "Livermore" models are based on the EPDL97, EADL public data libraries ${ }^{47-49}$ which include the required information for the determination of cross sections and for the description of the final state of each physical interaction. They are applicable to incident electrons and photons, covering elements from $\mathrm{Z}=1$ to 99 , from a few $10 \mathrm{~s}$ of eV up to $100 \mathrm{GeV}$, with a recommended low energy validity of $250 \mathrm{eV}$. Taking into account the shell structure of atomic elements, they are able to simulate atomic de-excitation processes such as fluorescence and Auger cascades. ${ }^{50}$ Electron-impact ionization is described by a continuous energy-loss model whereas the simulation of delta-ray production above a production-threshold (or "production-cut") is selected by the user. The theoretical calculation of electron-impact ionization cross sections is presented in the EEDL documentation: ${ }^{49}$ in summary, for close (or hard) collisions, Seltzer's modification of the Möller binary collision cross section is used, taking into account the binding of the electron in a given sub-shell, while for distant (or soft) collisions EEDL uses the modification of the Weizsacker-Williams method. ${ }^{49}$ Multiple scattering is not specific to the "Livermore" set of models and is simulated in the "Livermore" physics constructor using the Geant4 Urban model below $100 \mathrm{MeV} .{ }^{17,51}$ This model uses functions in order to calculate angular and spatial distribution after a step. These functions have been adapted in order to reproduce the same moments of the angular and spatial distributions of the Lewis theory (refer to the Geant4 Physics Reference Manual, accessible online at http://geant4.org).

\section{2. "Penelope" models}

The "Penelope" models derive from a re-engineering of the PENELOPE 2008 (Ref. 52) code and describe the interactions of electrons, positrons, and photons in atomic elements, from tens of $\mathrm{eV}$ up to $1 \mathrm{GeV}$, with a recommended low energy limit of $100 \mathrm{eV}$. Atomic de-excitation processes are considered for K, L, and M shells. Regarding electrons, like for the "Livermore" case, the ionization model computes the continuous energy-loss and simulates the production of secondary delta rays above a selected production-cut. The ionization model uses the Generalised Oscillator Strength (GOS) model by Liljequist ${ }^{53}$ and considers distant longitudinal collisions, distant transverse collisions, and close collisions. A further detailed description of this model is given in the Geant4 Physics Reference Manual (see http://geant4.org). Multiple scattering is also simulated in the "Penelope" physics constructor using the Geant4 Urban model below $100 \mathrm{MeV}$.

In Geant4, ionization is a discrete-continuous process which, along with multiple scattering and transportation, controls the step limitation (see Geant4 Physics Reference Manual: http://geant4.org). The settings for step limitation by ionization and multiple scattering are the same in both "Livermore" and "Penelope" physics constructors. However, the user has the possibility to force the maximum step size value of all simulation steps (using the so-called "G4StepLimiter" process) and we will use this feature in order to evaluate the influence of the step size on microdosimetry quantities.

\section{B. Geant4-DNA models}

In addition to the above-described two sets of (mixed) $\mathrm{CH}$ models (i.e., the "Livermore" and "Penelope" models), the Geant4-DNA low-energy extension of Geant4 includes sets of physics models that enable TS (i.e., step-by-step) simulation of charged-particle transport (e.g., electrons, protons and neutral hydrogen, alpha particles and their charge states, carbons, etc.) in liquid water down to the eV energy range. Regarding the simulation of electron interactions, users have the possibility to select three sets of alternative physics models which correspond to different cross sections for elastic and inelastic scattering. ${ }^{37}$ These physics models are: the default Geant4-DNA models, ${ }^{38}$ the models developed at the University of Ioannina (hereafter the "Ioannina" models ${ }^{54,55}$ ), and the CPA100 models, ${ }^{37,56}$ which will be released publicly in Geant4 in 2017. These three sets of models are assembled into Geant 4 physics constructors as summarized in Table I.

\section{Default option}

In the default option, the total and differential inelastic cross sections for the weakly bound electrons of liquid water are calculated numerically from the energy $(\hbar \omega)$ and momentum $(\hbar k)$ dependent complex dielectric response function, $\varepsilon(\omega, k)$, of the medium within the first Born approximation. Specifically, the optical-data model for $\varepsilon(\omega, k)$ of Emfietzoglou and co-workers, ${ }^{57-59}$ which considers four outer ionization shells $\left(1 b_{1}, 3 a_{1}, 1 b_{2}, 2 a_{1}\right)$ and five discrete electronic excitations ( $\mathrm{A}^{1} \mathrm{~B}_{1}, \mathrm{~B}^{1} \mathrm{~A}_{1}$, Ryd $\mathrm{A}+\mathrm{B}$, Ryd $\mathrm{C}+\mathrm{D}$, diffuse bands) of liquid water, is employed. In this model, the $\omega$-dependence of the dielectric function at $k=0$ is obtained by fitting the experimental data for both the real and imaginary parts of the dielectric function, $\varepsilon_{R}(\omega, k=0)$ and $\varepsilon_{I}(\omega, k=0)$, using a superposition of Drude-type functions with adjustable coefficients. Then, the energy-loss-function (ELF) at $k=0$ is calculated from $\operatorname{Im}\left[\frac{-1}{\varepsilon(\omega, k=0)}\right]=\frac{\varepsilon_{I}(\omega, k=0)}{\varepsilon_{R}^{2}(\omega, k=0)+\varepsilon_{I}^{2}(\omega, k=0)}$. To ensure selfconsistency, the f-sum-rule for $\varepsilon_{I}(\omega, k=0)$ and $\operatorname{Im}[-1 / \varepsilon(\omega, k=0)]$ is fulfilled to be better than $1 \%$. The partitioning of $\varepsilon_{I}(\omega, k=0)$, which is proportional to the optical oscillator strength, to the electronic absorption channels $(n)$ of liquid water, enables calculation of cross sections for each individual ionization shell and excitation transition, through $\frac{\varepsilon_{I}^{(n)}(\omega, k=0)}{|\varepsilon(\omega, k=0)|^{2}}$. These partial cross sections, which are essential to 
TABLE I. List of Geant4-DNA sets of electron cross section models used in this work. Stars indicate the corresponding physics constructors. Vibrational excitation and molecular attachment processes are not considered. Readers are invited to consult indicated references for more details on the physics models.

\begin{tabular}{lccc}
\hline \hline Set of cross sections & Default ${ }^{38 \mathrm{a}}$ & Ioannina ${ }^{54,55 \mathrm{~b}}$ & CPA100 ${ }^{37,56 \mathrm{c}}$ \\
\hline Ionization & $\begin{array}{c}\text { Emfietzoglou optical-data model of the } \\
\text { dielectric function with Born corrections }\end{array}$ & $\begin{array}{c}\text { Kyriakou-Emfietzoglou optical-data model } \\
\text { dielectric function with Born corrections }\end{array}$ & Relativistic Binary Encounter Bethe model \\
Excitation & Same & Same & $\begin{array}{c}\text { Differential oscillator strength from the } \\
\text { Dingfelder model of the dielectric response } \\
\text { function }\end{array}$ \\
Elastic & Partial-wave analysis & Rutherford formula with screening term by & Independent Atom Method \\
& Uehara et al. ${ }^{62}$ & \\
\hline \hline
\end{tabular}

aCorresponding Geant4-DNA physics constructor: G4EmDNAPhysics_option2 (abbreviated later as "option 2").

${ }^{\text {b}}$ Corresponding Geant4-DNA physics constructor: G4EmDNAPhysics_option4 (abbreviated later as "option 4").

${ }^{\text {cC}}$ Corresponding Geant4-DNA physics constructor: G4EmDNAPhysics_option6 (abbreviated later as "option 6").

TS simulation, provide a consistent description of secondary electron generation for both soft and hard collisions. To avoid an unphysical extrapolation of the inelastic cross sections down to zero $\mathrm{eV}$, the Drude functions are truncated at the various binding energies. Finally, to obtain the whole Bethe surface, the extension of $\varepsilon(\omega, k=0)$ to $k \neq 0$ is made by semiempirical dispersion relations for the Drude coefficients. Below a few hundred $\mathrm{eV}$, the first Born approximation is not directly applicable; a kinematic Coulomb-field correction and Mott-like exchange-correction terms are used. ${ }^{59}$

On the other hand, total and differential cross sections for electron-impact ionization of the K-shell (of the oxygen atom) are calculated analytically from the binary-encounterapproximation-with-exchange model (BEAX). ${ }^{60}$ This is an atomic model which depends only on the binding energy, the mean kinetic energy, and the occupation number of the orbital. The scattering angle of the primary electron and the ejection angle of the secondary electron in ionization events are determined from the kinematics of binary collisions.

No angular deflection is considered in collisions leading to electronic excitation. The elastic cross sections are based on partial wave (PW) calculations, considering a total interaction potential which takes into account a static contribution as well as fine effects, like exchange and polarization contributions. ${ }^{61}$ In this approach, no energy loss is considered to take place in elastic collisions.

\section{Ioannina option}

The Ioannina option provides updated electron cross sections for excitation and ionization, and an alternative elastic scattering model. ${ }^{54,55}$ For the calculation of inelastic cross sections, the starting point is the same Drude parameterization of $\varepsilon(\omega, k)$ used in the default option. Apart from its mathematical simplicity, the Drude model has the advantage that the Kramers-Kronig integrals can be solved analytically and that it fulfills the f-sum-rule independent of $k$. Thus, both $\varepsilon_{I}(\omega, k)$ and $\varepsilon_{R}(\omega, k)$ can be expressed analytically and the particular form of the dispersion relations for the $k$-dependence is to our disposal. However, a brute-force truncation of the Drude function (as done in the default model) violates the f-sum-rule and the expression for $\varepsilon_{R}(\omega, k)$ obtained from $\varepsilon_{I}(\omega, k)$ via the Kramers-Kronig relation becomes complicated. Both effects are not considered in the default option.
In the Ioannina option, the above problems are overcome through the implementation of an algorithm which redistributes $\varepsilon_{I}(\omega, k=0)$ to the individual inelastic channels in a physically motivated and f-sum-rule constrained manner. Thus, despite starting from essentially the same optical-data model for $\varepsilon(\omega, k)$, substantially different partial ELFs $\left(\frac{\varepsilon_{l}^{(n)}(\omega, k)}{|\varepsilon(\omega, k)|^{2}}\right)$ and, as a result, ionization and excitation cross sections are obtained by the Ioannina option. ${ }^{54}$ For example, excitations are strongly enhanced relative to ionizations (which decrease only moderately), resulting in higher Wvalues (defined as the average energy to produce an ion pair), smaller penetration distances, and less diffused dosepoint-kernels at sub-keV electron energies. ${ }^{54,55}$ In addition, methodological changes are made in the application of the Coulomb and Mott corrections which result in more accurate ionization cross sections, especially at energies near the binding energies. Finally, the elastic cross sections are calculated from the screened Rutherford (SR) formula using the screening parameter of Uehara et $a l .{ }^{62}$ which is deduced from a fit to experimental data for water vapor. Although less accurate at low energies than the PW calculations used in the default option, the use of an empirical screening parameter specific to water improves the performance of the simple SR model while enabling a fully analytical representation for both the total and differential cross section which is an advantage.

\section{CPA100 option}

In the case of CPA100 models, ${ }^{56}$ cross sections for electronic excitations are calculated in the first Born approximation using the optical-data model of $\varepsilon(\omega, k)$ developed by Dingfelder and co-workers. ${ }^{35}$ This model is also based on a Drude representation of $\varepsilon(\omega, k)$, using the same optical data set, electronic excitation levels, and dispersion relations with the default (and Ioannina) models. The resulting excitation cross sections, however, are not the same due to a different parameterization. The ionization cross sections for the five shells of water are calculated from the binary-encounterBethe (BEB) model. ${ }^{63}$ Thus, total and differential ionization cross sections are calculated analytically. Similar to the BEAX model, the BEB model is an exchange-corrected atomic model which depends only on the binding energy, the 


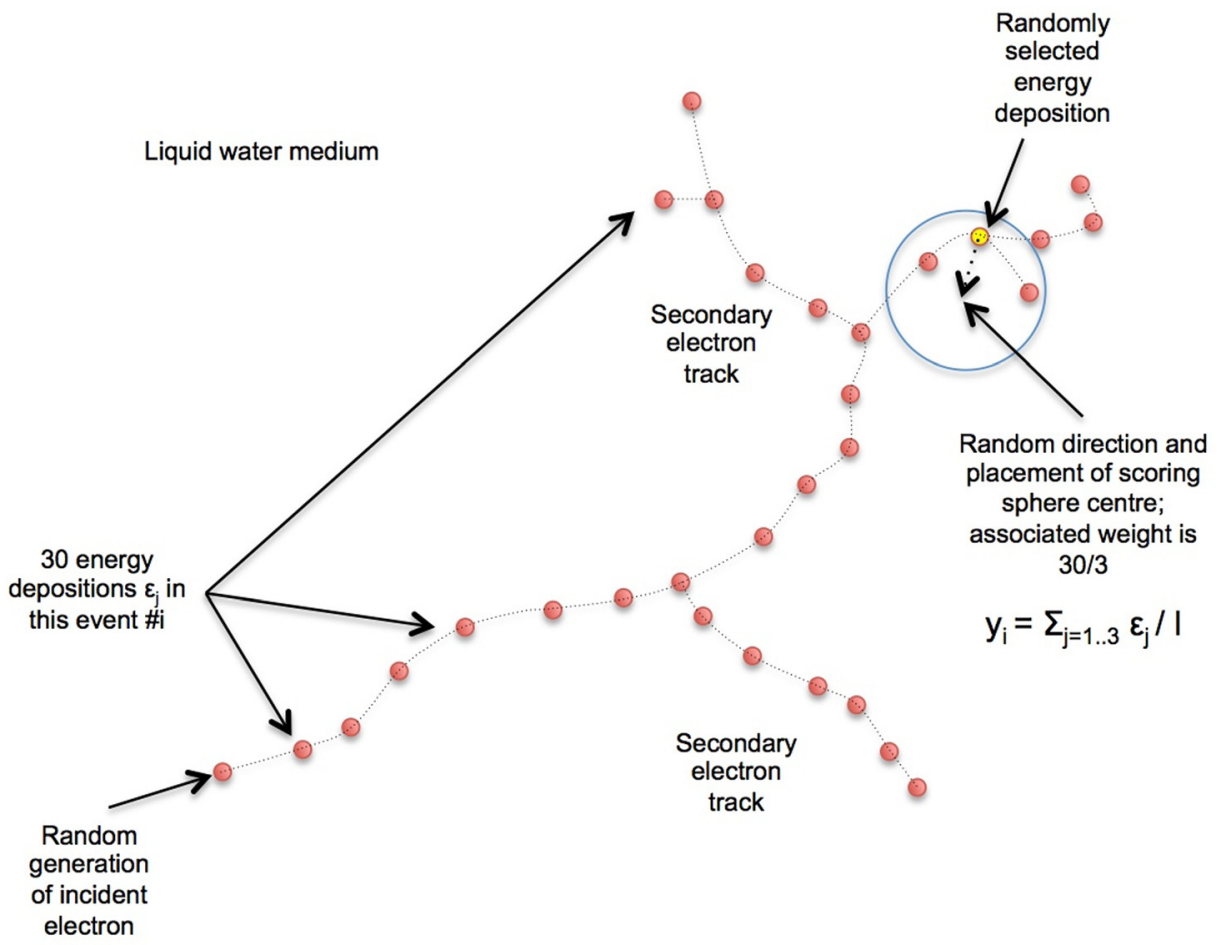

FIG. 1. Principle of scoring of lineal energy values (denoted as " $y_{i}$ ") for a single incident electron. The Geant 4 event illustrated in this figure contains 30 energy depositions (denoted as " $\varepsilon_{j}$," each being represented by a red disk). The scoring sphere of chord length 1 is represented by the blue circle. It is placed at a random distance (which is less or equal to the sphere radius) and in a random direction from a randomly selected energy deposition (represented by a yellow disk). In this illustration, the lineal energy is obtained by summing the three energy depositions contained in the sphere, and its associated statistical weight is taken as $30 / 3$.

mean kinetic energy, and the occupation number of the orbital. Angular deflections in both ionization and excitation collisions are considered based on the kinematics of binary collisions. Elastic scattering cross sections are based on PW calculations using the independent atom approximation. Two variants of elastic scattering were studied: one case where the elastic scattering process is not accompanied by a kinetic energy loss, and another one when this energy loss is taken into account (this is the default setting of the CPA100 elastic model in the original CPA100 code $^{56}$ ). This small energy
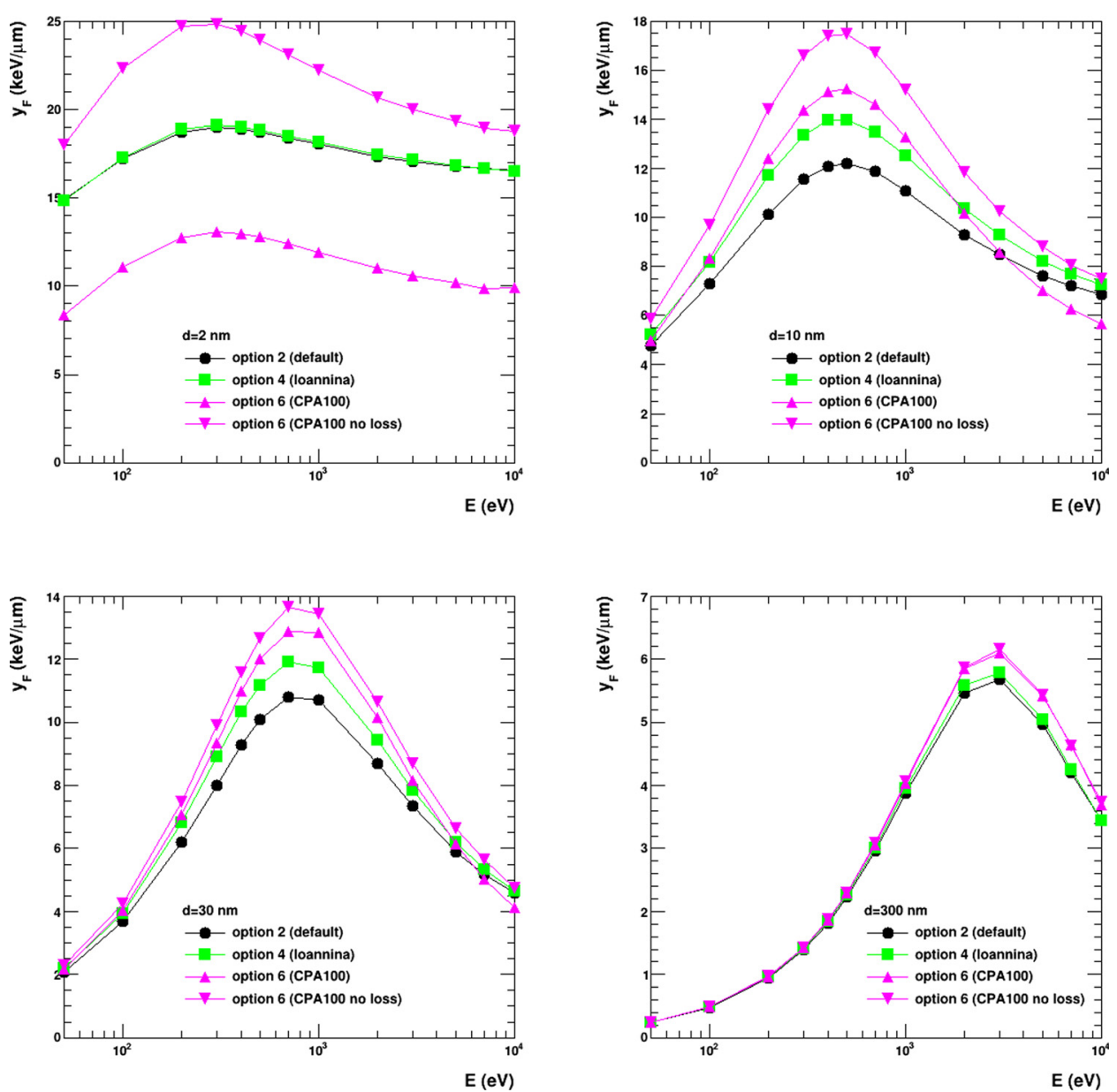

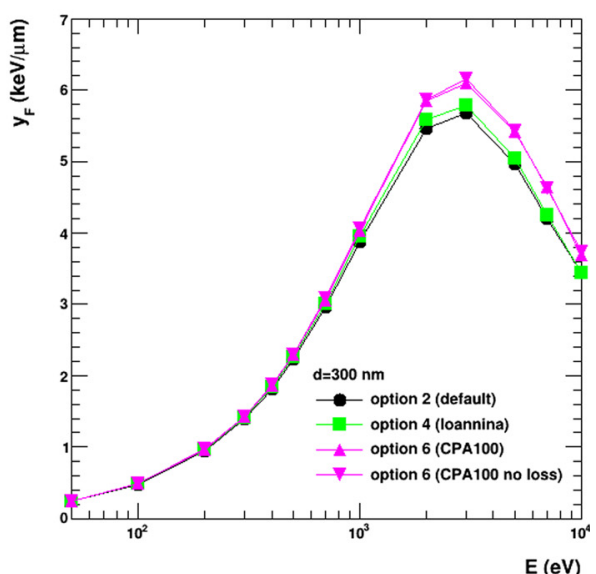

FIG. 2. Frequency-mean lineal energy as a function of incident electron kinetic energy, in scoring spheres of $2 \mathrm{~nm}$ diameter (top left plot), $10 \mathrm{~nm}$ (top right plot), $30 \mathrm{~nm}$ (bottom left plot), and $300 \mathrm{~nm}$ (bottom right plot). The curves correspond to the different Geant4-DNA physics constructors (see Table I): Black circles: "option 2" constructor (default models); Green squares: "option 4" constructor (Ioannina models); Purple up-triangles: "option 6" constructor (CPA100 models) with energy-loss in elastic scattering activated; Purple down-triangles: "option 6" constructor (CPA100 with de-activated energy-loss in elastic scattering). 
loss derives from energy-momentum conservation constraints during the elastic scattering process (due to nuclei recoil) and is usually neglected by track-structure codes which simulate electrons interactions in liquid water (for more details see Sec. 2.1.1 of Ref. 56).

\section{Sampling}

The definitions and formalism of the microdosimetric quantities used in this work are presented in detail in the Appendix. We describe below the different approaches used in the simulations in order to calculate the $y$ and $z$ distributions.

\section{Lineal energy $y$}

The probability density function of lineal energy is obtained from the scoring of all energy depositions occurring in the target (or probe) volume. For a selected Geant4-DNA physics constructor, tracks of incident particles (single events) are simulated one-by-one in a large volume of liquid water. Geant4 hit collections are used in order to record for each single event all energy depositions and their location. The radius of the probe spherical volume is chosen by the user. Once the interactions of an incident particle (single event) including all its secondary particles have all been simulated, we randomly sample one energy deposition ("hit") and the probe volume is randomly placed at a distance from this hit less than the probe radius; all energy depositions corresponding to hits located within the probe volume are then added to $\varepsilon$. The value of $\varepsilon$ is then weighted with a ratio equal to the total number of hits divided by the number of hits located in the probe volume, in order to ensure a "spatially uniform sampling." Such a weighting indeed prevents bias towards regions of high density of hits. ${ }^{1}$

The lineal energy value is obtained from the ratio $y=\varepsilon / \bar{l}$, where $\bar{l}$ is the mean chord length of the probe volume (see the Appendix). Figure 1 illustrates the principle of scoring of lineal energy values for a single incident electron.

The procedure is then repeated for many other independent single events in order to improve the statistical accuracy of the results. Once calculated, the frequency-mean lineal energy $\bar{y}_{F}$ can be extracted. The dose probability density function is directly obtained using the relation

$$
d(y)=y f(y) / \bar{y}_{F} .
$$

\section{Specific energy $z$}

The exact same procedure is adopted for the scoring of $z$ values, which are calculated as the ratio $z=\varepsilon / m$. The dose probability density function of specific energy normalized to a single event is directly obtained from

$$
d_{1}(z)=z f_{1}(z) / \bar{z}_{1 F},
$$

where $\bar{z}_{1 F}$ is the frequency-mean specific energy.
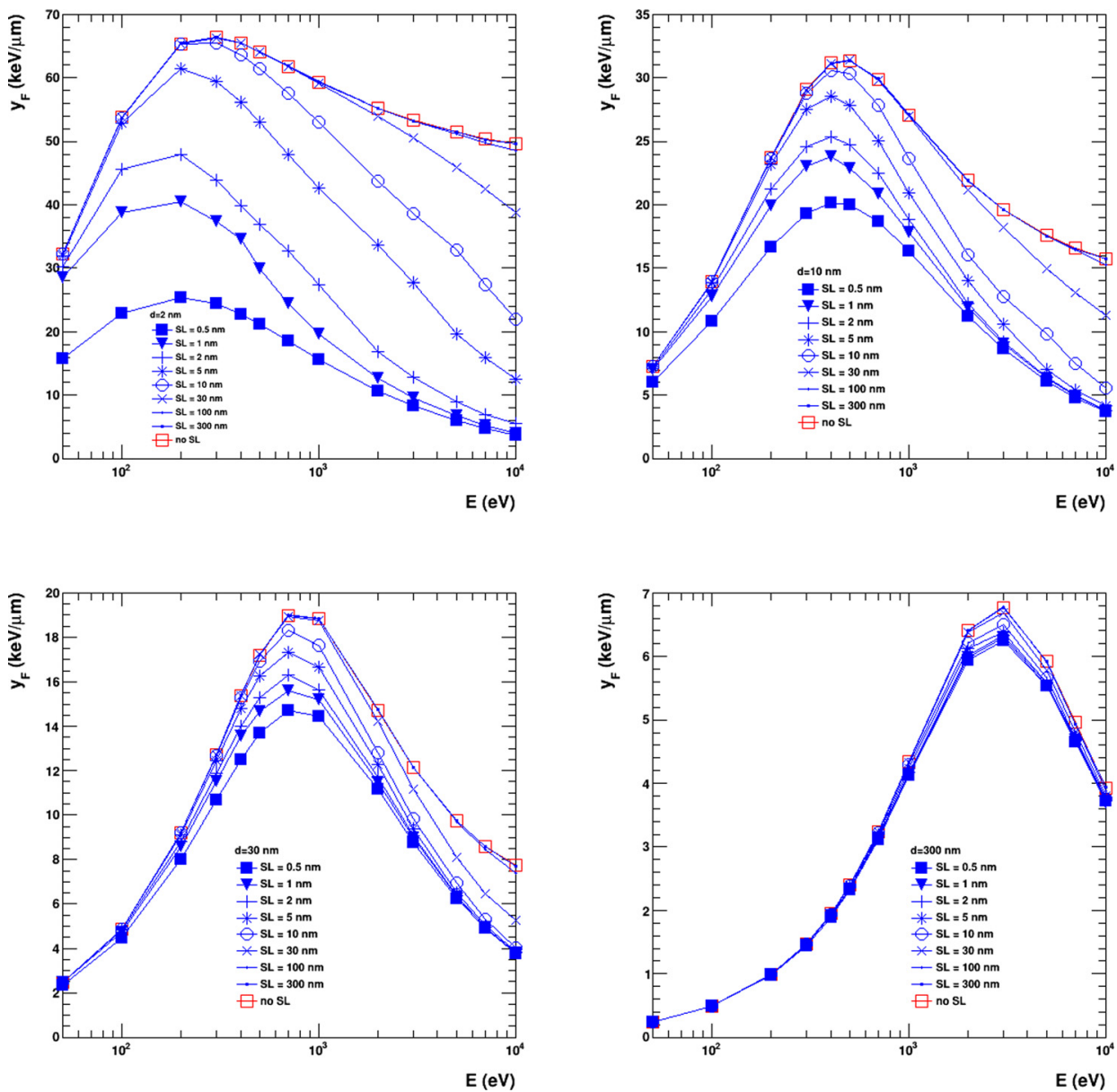

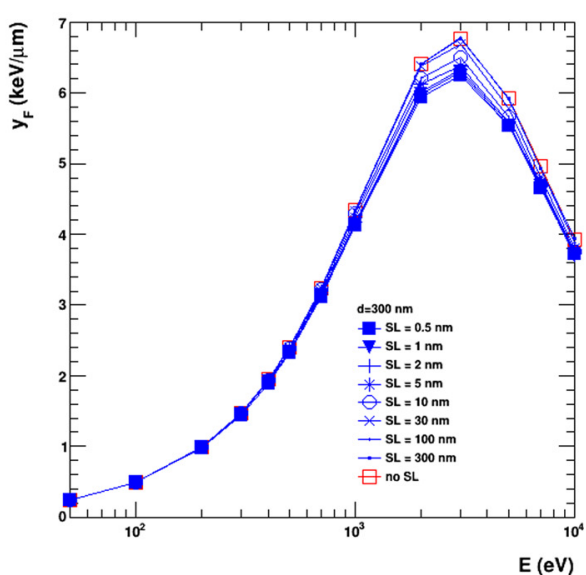

FIG. 3. Frequency-mean lineal energy as a function of incident electron kinetic energy, in scoring spheres of $2 \mathrm{~nm}$ diameter (top left plot), $10 \mathrm{~nm}$ (top right plot), $30 \mathrm{~nm}$ (bottom left plot), and $300 \mathrm{~nm}$ (bottom right plot). The curves are obtained using the "Livermore" physics constructor for electrons, for different step-size limits (SL): Red open-squares: no SL; Blue filledsquares: $\mathrm{SL}=0.5 \mathrm{~nm}$; Blue down-triangles: $\mathrm{SL}=1 \mathrm{~nm}$; Blue plus-symbols: $\mathrm{SL}=2 \mathrm{~nm}$; Blue stars: $\mathrm{SL}=5 \mathrm{~nm}$; Blue open-circles: $\mathrm{SL}=10 \mathrm{~nm}$; Blue crosses: $\mathrm{SL}=30 \mathrm{~nm}$; Blue small plus-symbols: $\mathrm{SL}=100 \mathrm{~nm}$; Blue small-squares: $\mathrm{SL}=300 \mathrm{~nm}$. A secondary productioncut of $11 \mathrm{eV}$ is applied in all cases. 


\section{Normalization}

Microdosimetric distributions are usually represented using logarithmic (along abscissae) and linear (along ordinates) discrete histogram plots: these probability density functions defined previously are multiplied by lineal energy values $(y)$ or by specific energy values $(z)$ and are shown as a function of $\log$ values of $y$ or $z$. This allows for the visualization of the contribution of $y$ or $z$ over a large range of values. The area under the curve $y f(y)$ (or $z f(z)$ ) between two values of $y$ (or $z$ ) is proportional to the fraction of events in this range. Similarly, the area under the curve $y d(y)$ (or $z d_{1}(z)$ ) between two values of $y$ (or $z$ ) is proportional to the fraction of dose by events with lineal energy (or specific energy) in this range. Such semi-log representation requires specific normalization as explained by several authors: ${ }^{64,65}$ for example, the normalization of $f(y)$ is obtained from

$$
\begin{aligned}
\int_{0}^{\infty} f(y) d y & =\int_{0}^{\infty} y f(y) d(\ln y)=\ln 10 \int_{0}^{\infty} y f(y) d\left(\log _{10} y\right) \\
& \approx \frac{\ln 10}{B} \sum_{i=0}^{\infty} y_{i} f\left(y_{i}\right)=1,
\end{aligned}
$$

where $B$ represents the number of histogram bins per decade and $i$ runs over all histograms bins.

\section{E. The "microyz" Geant4 extended example}

All simulation results described in this work are based on the "microyz" Geant4 extended example, which will be released soon in Geant4 after publication of this work. This example aims to explain how to simulate microdosimetry spectra ( $y$ and $z$ distributions) as well as microdosimetry quantities $\left(\bar{y}_{F}, \bar{y}_{D}, \bar{z}_{F}, \bar{z}_{D}\right)$ (cf. Appendix) using Geant4-DNA physics models and can be run in multithreading mode. These quantities are computed using ROOT ${ }^{66}$ macros. In addition, the user has the possibility to fully drive the simulation using User Interface commands, for the selection of, Geant4-DNA physics constructor, tracking-cut, incident particle type, and incident energy. A tracking-cut of $11 \mathrm{eV}$ has been applied to all electron simulations, that is, all electrons with kinetic energy below this value are stopped and their kinetic energy is released locally into the liquid water medium. This is the minimum energy limit of applicability of CPA100 models, while the default Geant4-DNA models have a minimum energy limit of $7.4 \mathrm{eV}$ and the Ioannina models have a minimum energy limit of $9 \mathrm{eV}$; for a proper comparison, the same tracking-cut of $11 \mathrm{eV}$ was therefore applied to all three simulation options. Vibrational excitation and molecular attachment processes for electrons have not been considered, since such processes are only available for the default Geant4DNA set of physics models. Atomic de-excitation ${ }^{50}$ (that is, emission of fluorescence photons and Auger electrons) has been activated in all simulations.
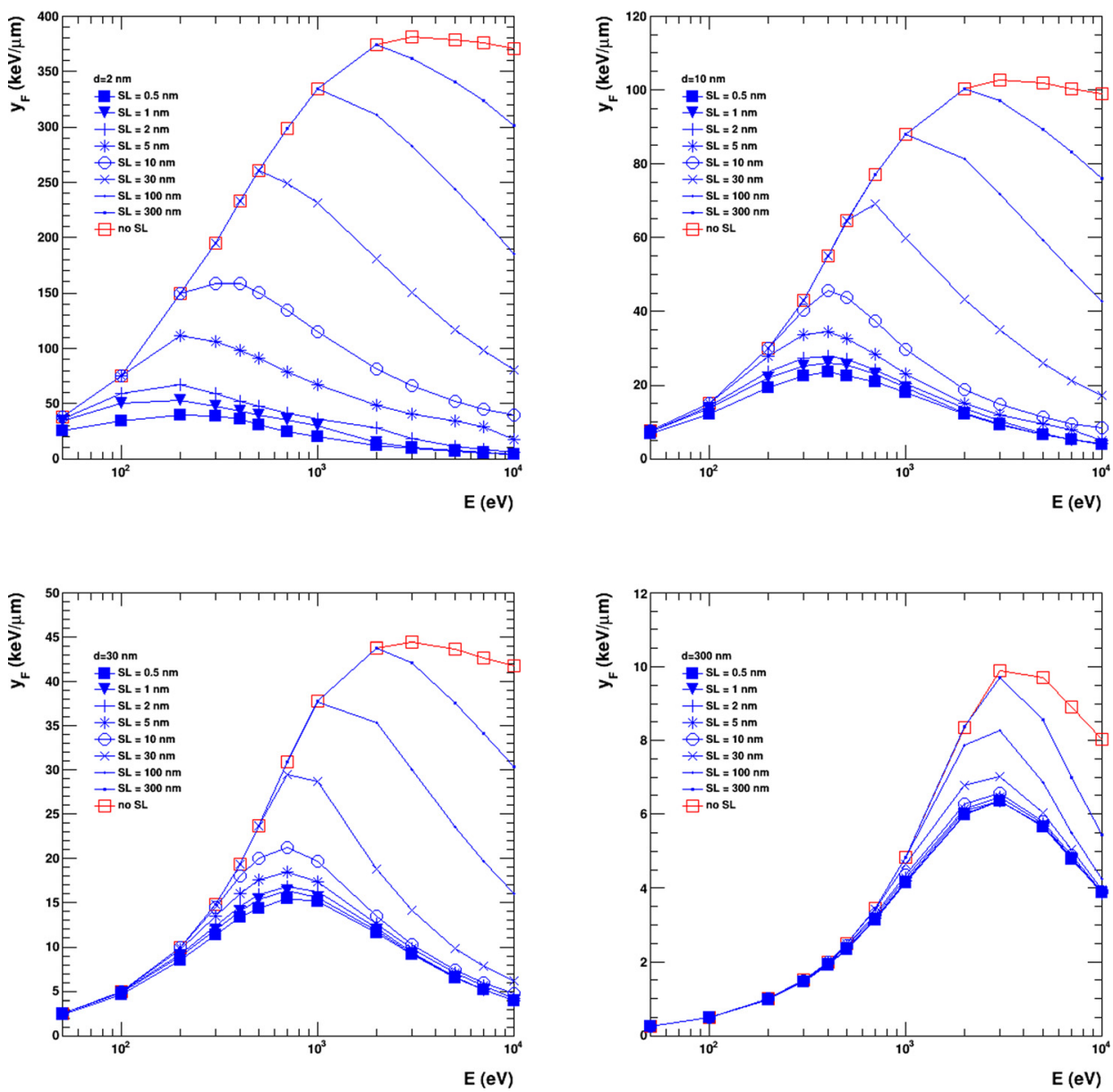

FIG. 4. Same as Fig. 3 for the "Penelope" physics constructor. 
Note that the results presented in this work for the "Livermore" and "Penelope" models are obtained using the "G4EmLivermorePhysics" and "G4EmPelenopePhysics" physics constructors provided to Geant 4 users for low-energy electromagnetic physics simulations. We did not investigate the tuning of the physics model parameters, as set by default in these constructors (such as step size limitation parameters for the continuous-discrete ionization or for continuous multiple scattering processes), but we instead investigated the influence of secondary particle production-cut (expressed as an energy) and maximum step size limit (expressed as a distance, using the "G4StepLimiter" process), in comparison to TS Geant4-DNA simulations. The sensitivity of the results to these specifications is examined in detail. In addition, when using the "G4EmLivermorePhysics" and "G4EmPelenopePhysics" physics constructors, and in order to be consistent with the tracking-cut used for Geant4-DNA simulations, the lowest energy for tracking electrons (available since Geant 4 release 10.2) was set to $11 \mathrm{eV}$ (the default value is $100 \mathrm{eV}$ ).

All simulations in the present work were performed using Geant4 10.2.P01 (February 2016) on an 80-core 64 bits Scientific Linux cluster. They can also run using for example a multi-core virtual machine; such a virtual machine and future upgrades are freely downloadable from http://geant4.in2p3.fr.

\section{RESULTS AND DISCUSSION}

In total, 13 incident electron energies were examined: 50, $100,200,300,400,500,700 \mathrm{eV}, 1,2,3,5,7$, and $10 \mathrm{keV}$, the latter being the maximum energy limit of applicability of the "Ioannina" physics models. An incident statistics of $10^{6}$ electrons was selected for incident energies up to $5 \mathrm{keV}$, and $10^{5}$ above $5 \mathrm{keV}$, which allows maintaining a statistical fluctuation for $\bar{y}_{F}$ (or $\bar{z}_{1 F}$ ) and $\bar{y}_{D}\left(\right.$ or $\bar{z}_{1 D}$ ) below about $1 \%$ without exceedingly long simulation times. Scoring spheres with diameters relevant to sub-cellular biological targets were chosen: DNA base pair $(2 \mathrm{~nm})$, nucleosome $(10 \mathrm{~nm})$, chromatin fiber $(30 \mathrm{~nm})$, and chromosome $(300 \mathrm{~nm})$.

\section{A. Frequency-mean lineal energy $\bar{y}_{F}$}

Frequency-mean lineal energy $\left(\bar{y}_{F}\right)$ for the three Geant4DNA physics constructors (default, Ioannina, CPA100) as a function of incident energy (with or without energy loss during elastic scattering for CPA100) and for different scoring sphere diameters is shown in Fig. 2. Figures 3 and 4 show the corresponding distributions for the low-energy EM physics constructors "Livermore" and "Penelope," respectively, and for different maximum step-size limits (assuming a secondary production-cut of $11 \mathrm{eV}$ ). Finally, Figs. 5 and 6 show the corresponding distributions for the "Livermore" and "Penelope" physics constructors, respectively, when different secondary production-cuts are chosen (no maximum step-size limit is applied).

Geant4-DNA results obtained with any of the three physics constructors (default, Ioannina, CPA100) are based on TS simulations (i.e., event-by-event). As a general observation, the Geant4-DNA distributions from the different
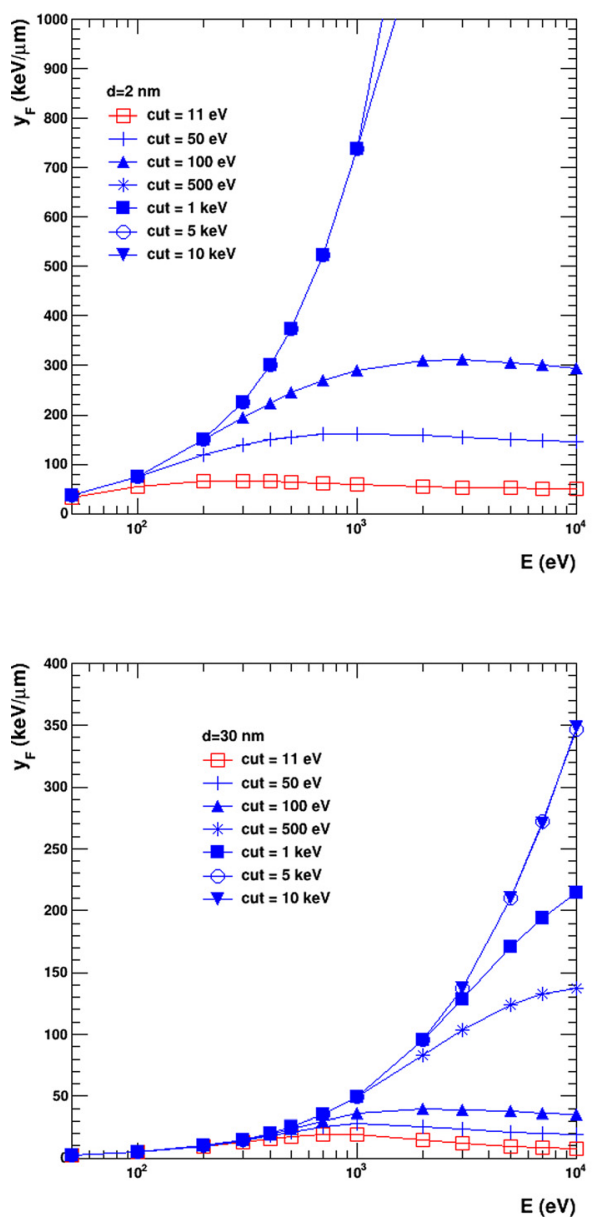
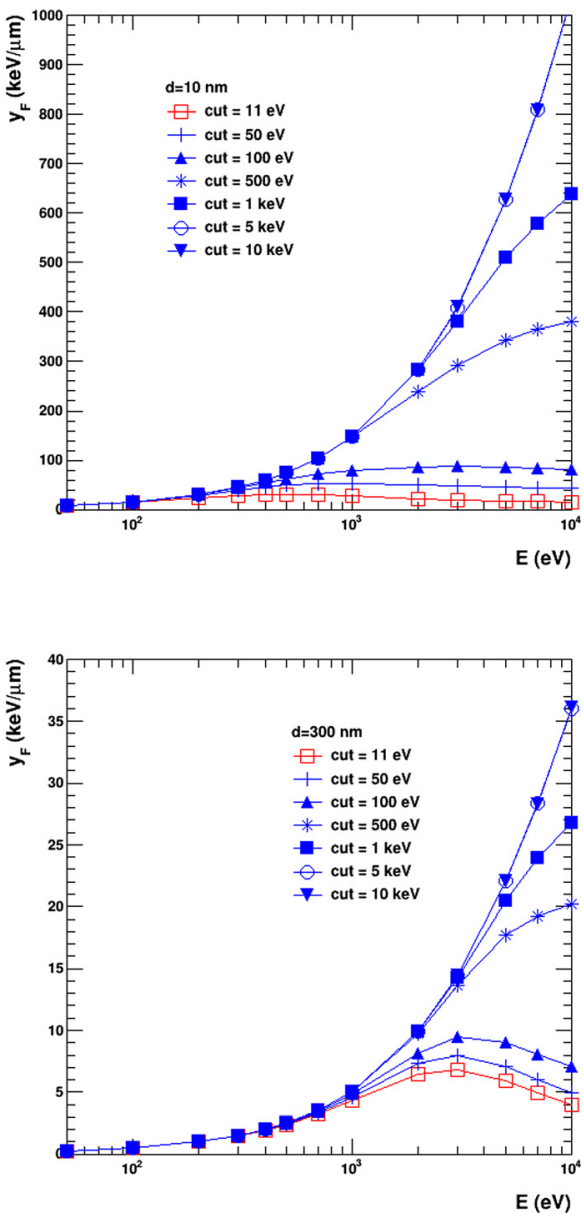

FIG. 5. Frequency-mean lineal energy as a function of incident electron kinetic energy, in scoring spheres of $2 \mathrm{~nm}$ diameter (top left plot), $10 \mathrm{~nm}$ (top right plot), $30 \mathrm{~nm}$ (bottom left plot), and $300 \mathrm{~nm}$ (bottom right plot). The curves are obtained using the "Livermore" physics constructors for different production-cuts: Red opensquares: $11 \mathrm{eV}$ cut; Blue crosses: $50 \mathrm{eV}$ cut; Blue up-triangles: $100 \mathrm{eV}$ cut; Blue stars: $500 \mathrm{eV}$ cut; Blue full-squares: $1 \mathrm{keV}$ cut; Blue open-circles: $5 \mathrm{keV}$ cut; Blue down-triangles: $10 \mathrm{keV}$ cut. No step-size limitation has been applied in all cases. Note that for the top left plot, blue squares $(1 \mathrm{keV})$, open circles $(5 \mathrm{keV})$ and blue down-triangles $(10 \mathrm{keV})$ are not distinguishable. 

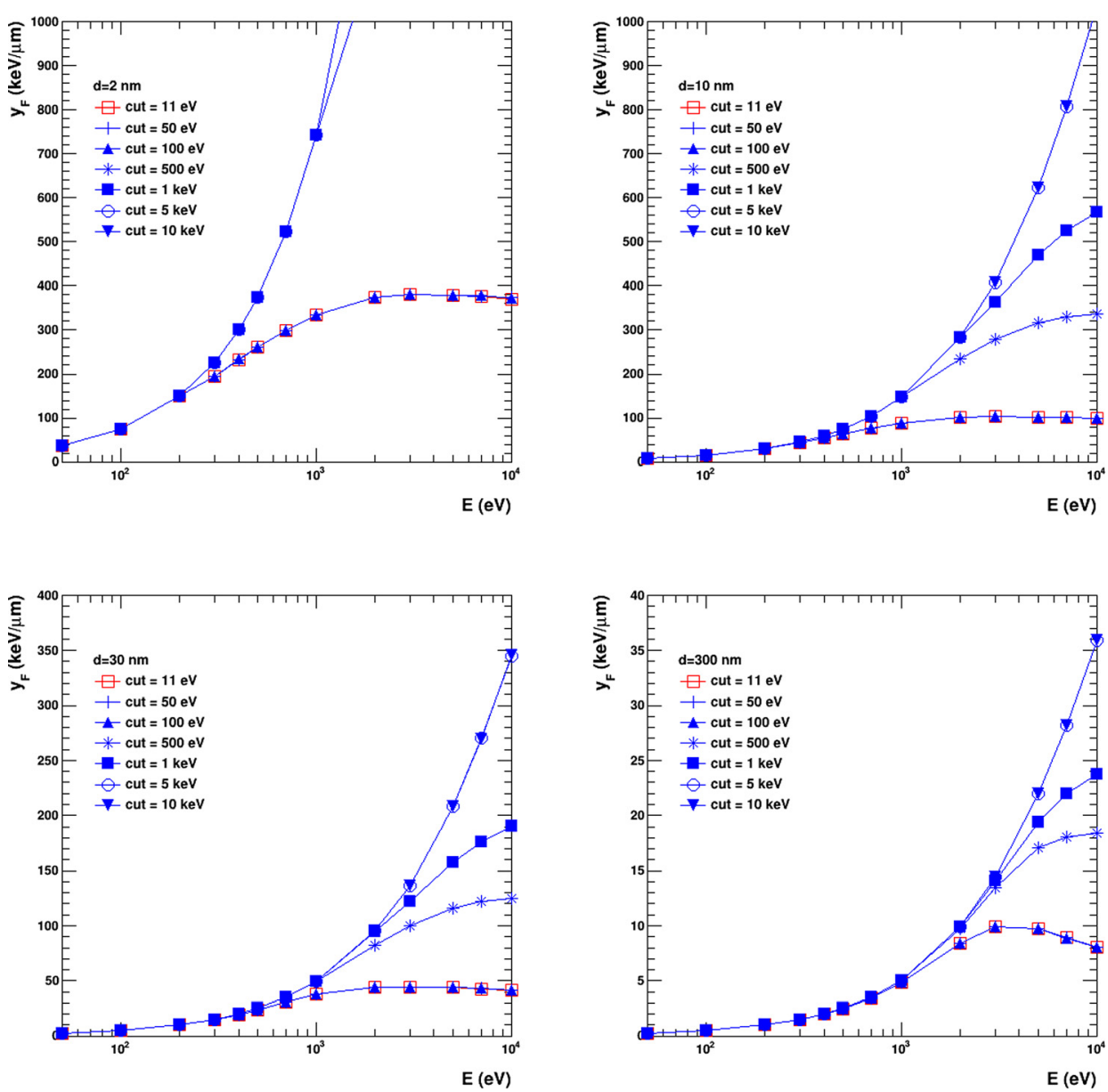

FIG. 6. Same as in Fig. 5 for the "Penelope" physics constructor. Note that for the top left plot, blue stars $(500 \mathrm{eV})$, blue squares $(1 \mathrm{keV})$, open circles $(5 \mathrm{keV})$, and blue downtriangles $(10 \mathrm{keV})$ are not distinguishable. physics constructors show a similar trend as a function of incident energy, and the larger the scoring volume the closer the agreement. For example, independent of the physics constructor used, the maximum of $\bar{y}_{F}$ is found at $\sim 300 \mathrm{eV}$ for the $2 \mathrm{~nm}$ sphere, at $\sim 500 \mathrm{eV}$ for the $10 \mathrm{~nm}$ sphere, at $\sim 700 \mathrm{eV}$ for the $30 \mathrm{~nm}$ sphere, and at $\sim 3 \mathrm{keV}$ for the $300 \mathrm{~nm}$ sphere. The default ("option 2") and Ioannina ("option 4") constructors are in very good agreement for the $2 \mathrm{~nm}$ and $300 \mathrm{~nm}$ spheres, but less so for the $10 \mathrm{~nm}$ and $30 \mathrm{~nm}$ spheres where differences are noticeable (although still small) reaching up to about $16 \%$ for the $10 \mathrm{~nm}$ case and up to $11 \%$ for the $30 \mathrm{~nm}$ case. The overall fair agreement between these two physics constructors is not surprising since their inelastic mean free paths are similar, differing mainly on the relative contribution of ionizations and excitations. ${ }^{37}$ On the other hand, the agreement points out to the small effect of the different elastic scattering models used in the two constructors (PW in "option 2" versus SR in "option 4"). Larger deviations are observed with the CPA100 ("option 6") constructor which compares poorly with the results from the default ("option 2") and Ioannina ("option 4") constructors for the $2 \mathrm{~nm}$ sphere, moderately for the $10 \mathrm{~nm}$ and $30 \mathrm{~nm}$ spheres, and fairly good for the $300 \mathrm{~nm}$ sphere. These deviations are mainly a consequence of the much different inelastic cross section models used in CPA100 (Sec. II B 3) compared to the default (Sec. II B 1) and Ioannina (Sec. II B 2) constructor as explained above (also consult Figs. 2 and 4 of Ref. 37). Regarding CPA100, we may also note the strong influence of the activation or inactivation of energy-loss during elastic scattering (except for the largest sphere where the effect is negligible). The use of elastic scattering without energy-loss systematically leads to larger frequency-mean lineal energies compared to the case where such energy losses are ignored. This is because elastic scattering is associated with very small energy losses given by $\mathrm{E}(1-\cos \theta) \times 1.214 \times 10^{-4}$ (where $\mathrm{E}$ is the electron incident kinetic energy and $\theta$ is the electron elastic scattering angle, see Ref. 56). Such very small energy losses tend to decrease the frequency-mean lineal energy when all possible values of lineal energy are averaged over the total number of simulated electrons. For completeness, we also investigated the distribution of frequency-mean lineal energy for the CPA100 models including energy loss during elastic scattering and calculated by scoring energy loss due to inelastic processes (electronic excitation and ionization) only; since energy losses during elastic scattering are very small, the values of $\bar{y}_{F}$ are quasi identical to the case where energy loss during elastic scattering is not taken into account (not shown).

Geant 4 results obtained using the low-energy EM constructors ("Livermore" and "Penelope") are based on (mixed) $\mathrm{CH}$ simulations. These simulations depend strongly on the maximum step-size limit and secondary productioncut values. In Fig. 3 ("Livermore") and Fig. 4 ("Penelope"), the effect of the maximum step-size limit to the frequencymean lineal energy is examined (fixed production-cut at $11 \mathrm{eV}$ is used). In all cases, "Penelope" values are larger than 

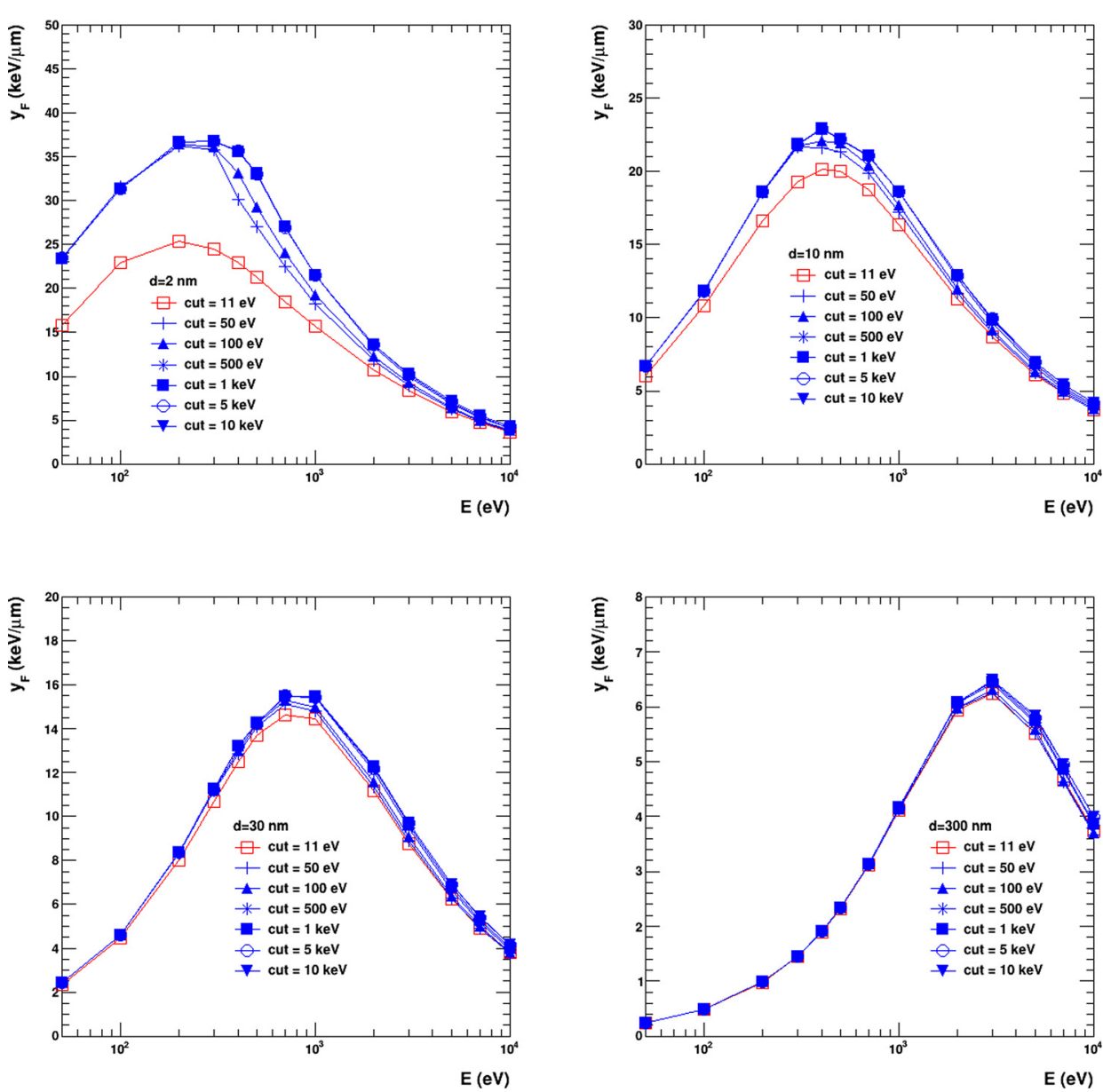

FIG. 7. Same as Fig. 5 for the "Livermore" physics constructor, applying a step-size limitation of $0.5 \mathrm{~nm}$.
"Livermore" values, and the results of both are systematically larger than those of Geant4-DNA. This is because $\mathrm{CH}$ simulations tend to overestimate the energy deposition in small volumes compared to TS simulations, owing to incomplete energy-loss straggling and the approximation that the total energy-loss from multiple inelastic collisions is deposited at a single point. As the step-size limit is reduced, the results from "Livermore" and "Penelope" constructors tend to approach the values obtained with Geant4-DNA (values smaller than $0.5 \mathrm{~nm}$, not shown, did not significantly improve the results but required much longer computation times). A comparison with the results of Fig. 2 reveals that, for the smallest step-size limit $(0.5 \mathrm{~nm})$, the agreement between the $\mathrm{CH}$ simulations ("Penelope" and "Livermore") and the TS simulations (Geant4-DNA) is good for the $300 \mathrm{~nm}$ sphere, moderate for the $30 \mathrm{~nm}$ sphere, and rather poor for the $10 \mathrm{~nm}$ and $2 \mathrm{~nm}$ spheres. Setting no step-size limitation in the "Penelope" and "Livermore" constructors gives the worst results.

In Fig. 5 ("Livermore") and Fig. 6 ("Penelope"), the effect of the secondary production-cut to the frequencymean lineal energy is examined (no step-size limit is used). The smallest production-cut value examined is $11 \mathrm{eV}$, compatible with the minimum kinetic energy down to which electrons are tracked in the present simulations (taken as $11 \mathrm{eV}$ ). A comparison with the Geant4-DNA results (see Fig. 2) reveals that, for both "Penelope" and "Livermore," reducing the production-cut improves the agreement with Geant4DNA. Note that in the case of the "Penelope" constructor, production-cuts equal to or lower than $100 \mathrm{eV}$ lead to identical curves since this value $(100 \mathrm{eV})$ is considered in Geant4 as the low energy limit of validity of the "Penelope" models.

Finally, Figs. 7 ("Livermore") and 8 ("Penelope") present the effect of the secondary production-cut on frequencymean lineal energy distributions, in the case where a step-size limitation of $0.5 \mathrm{~nm}$ is applied. When such a strong step-size limitation is applied, mimicking a TS (event-byevent) approach, distributions are much less dependent on production cut values, especially for the large diameters. The dependence appears slightly more pronounced with the "Livermore" models than with the "Penelope" models. The distributions remain globally closer to Geant4-DNA values shown in Fig. 2. In particular, both sets ("Livermore" and "Penelope") are slightly larger than Geant4-DNA distributions for the $2 \mathrm{~nm}, 10 \mathrm{~nm}$, and $30 \mathrm{~nm}$ diameters, and become similar for $300 \mathrm{~nm}$. However, we must add that such simulations with a strong step-size limitation induce significant computing time penalty (of at least two orders of magnitude), compared to the case where no step-size limitation is applied.

All these observations clearly support the necessity to adopt a TS (event-by-event) approach (i.e., Geant4-DNA) to avoid any dependence of microdosimetry results on step-size and production-cut values (necessary to $\mathrm{CH}$ simulations) which have to be set as low as possible for obtaining realistic results with the low-energy EM constructors ("Livermore," "Penelope") of Geant4. 

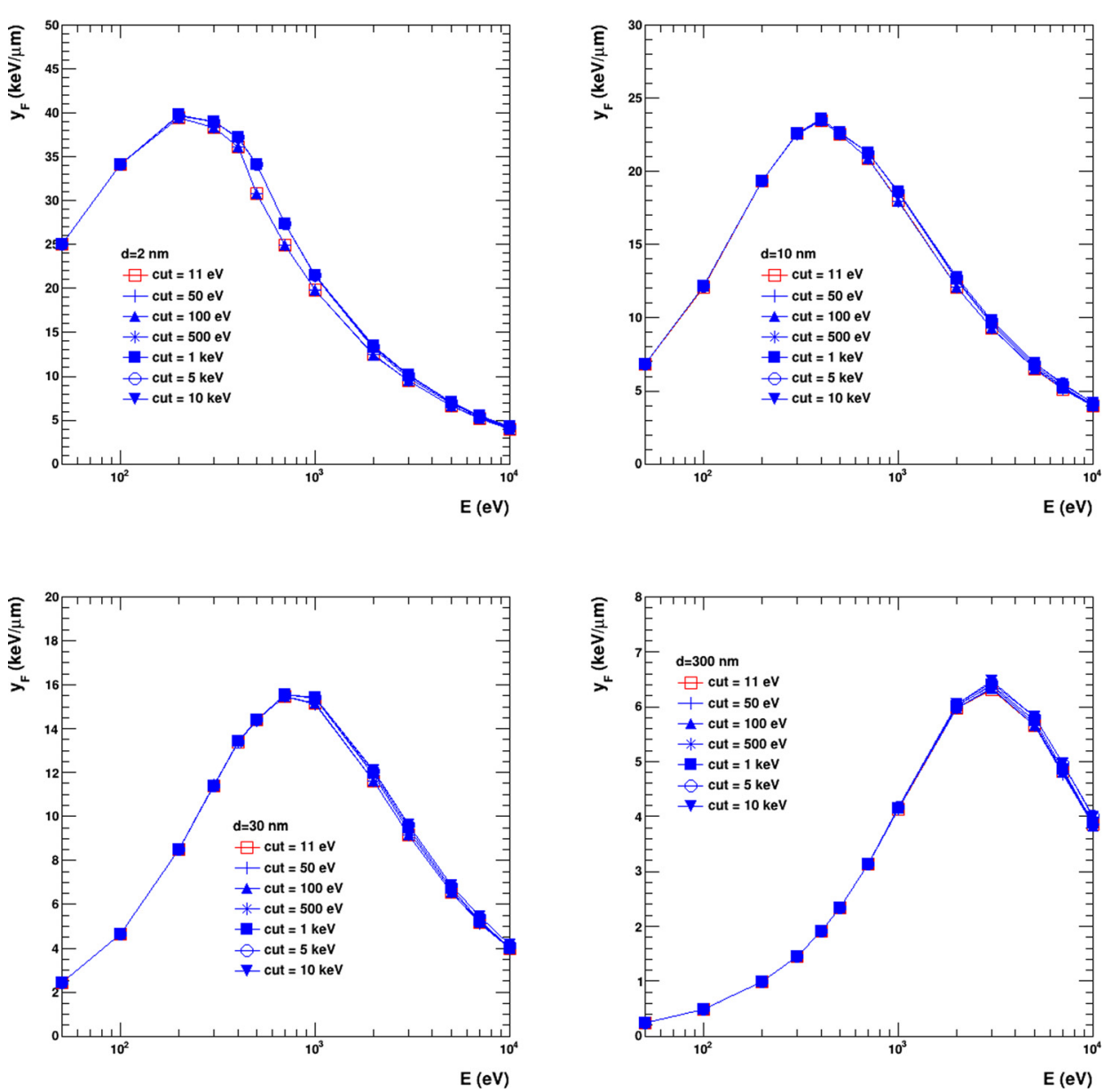

FIG. 8. Same as Fig. 6 for the "Penelope" physics constructor, applying a step-size limitation of $0.5 \mathrm{~nm}$.
Note that, for a spherical site of a unit density material, the frequency-mean lineal energy $\left(\bar{y}_{F}\right)$ depicted in Figs. 2-8 can be directly translated to frequency-mean specific energy for single-events $\left(\bar{z}_{1 F}\right)$ via the relation $z=0.204 \frac{y}{d^{2}}$ where $d$ must be in $\mu \mathrm{m}, y$ in $\mathrm{keV} / \mu \mathrm{m}$, and $z$ in Gy.

\section{B. Dose-mean lineal energy $\bar{y}_{D}$}

The dose-mean lineal energy $\left(\bar{y}_{D}\right)$ represents the lineal energy with which, on average, the dose is delivered by radiation in the site of interest. ${ }^{67}$ Dose-mean lineal energies for the three Geant4-DNA physics constructors (default, Ioannina, CPA100) and the "Livermore" and "Penelope" constructors, as a function of incident energy and for different scoring sphere diameters, are shown in Fig. 9. For the $\mathrm{CH}$ simulations ("Livermore" and "Penelope"), a step-size of $0.5 \mathrm{~nm}$ and a production-cut of $11 \mathrm{eV}$ have been used, since they were found in the previous section to yield the best agreement with the Geant4-DNA results (see Sec. III A). Note that the presence or absence of energy-loss during elastic scattering in the CPA100 constructor has no visible effect on $\bar{y}_{D}$ obtained in "option 6." This is a direct consequence of the very small energy losses occurring during the elastic process. Indeed, the dose-mean lineal energy is calculated from the integration of the product $y d(y)$ over $y$; thus, it is not significantly affected by such small contributions. The distributions depicted in Fig. 9 exhibit the same dependence with incident energy as in Fig. 2 and similar conclusions can be drawn. Specifically, the default ("option
2") and Ioannina ("option 4") constructors are, overall, in good agreement, while the results of the CPA100 ("option 6") are clearly larger for all scoring spheres examined with the differences becoming more pronounced with decreasing sphere diameter. As expected from the results presented in Sec. III A, both the "Livermore" and "Penelope" constructors yield $\bar{y}_{D}$ values that are larger than Geant4-DNA (independent of the physics models used) with the difference decreasing with sphere size. It is also clear that the "Livermore" results are closer to Geant4-DNA than "Penelope." In particular, the agreement between "Livermore" and CPA100 is fairly good even for the smallest sphere ( $2 \mathrm{~nm}$ diameter).

Similarly to the frequency-mean results, the doseweighted lineal energy $\left(\bar{y}_{D}\right)$ presented in Fig. 9 may be translated to dose-weighted specific energy for single-events $\left(\bar{z}_{1 D}\right)$ via the relation $z=0.204 \frac{y}{d^{2}}$ (see the Appendix).

Figures $10-13$ present calculated $y d(y)$ distributions as a function of $y$ for $1 \mathrm{keV}$ incident electrons for all the examined physics constructors and scoring spheres. The results are presented in logarithmic $\mathrm{x}$-scale and linear $\mathrm{y}$-scale, after normalisation (as discussed in Sec. II D). The average values of these distributions correspond to the $\bar{y}_{D}$ values shown in Fig. 9. Regarding Geant4-DNA distributions, the sharp vertical peaks observed on spectra correspond to discrete energy losses (and their combination) associated with the transition energies of the excitation levels and the binding energies of the ionization shells, occurring when incident and secondary electrons excite or ionize liquid water molecules (we recall 

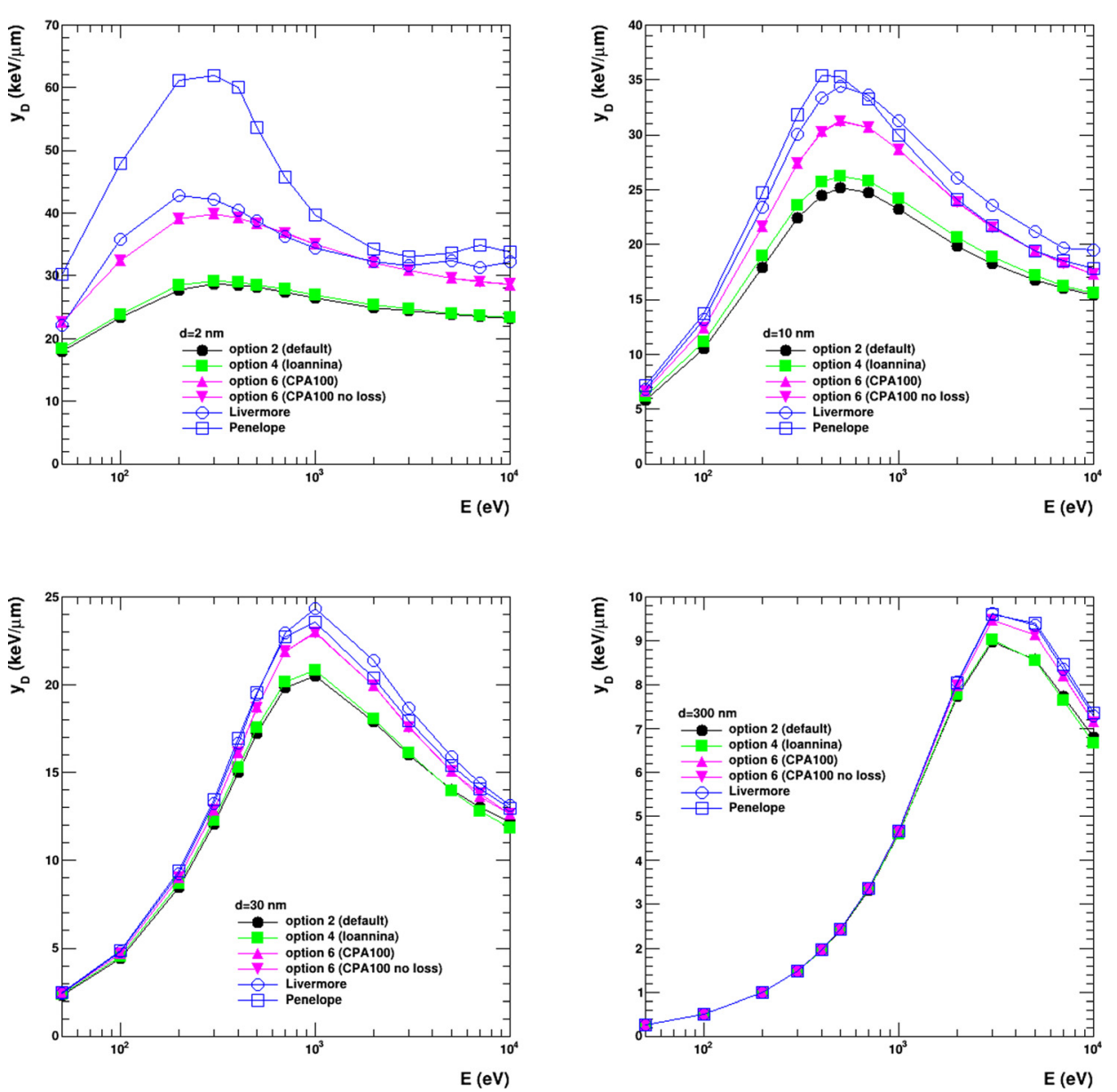

FIG. 9. Dose-mean lineal energy as a function of incident electron kinetic energy, in scoring spheres of $2 \mathrm{~nm}$ diameter (top left plot), $10 \mathrm{~nm}$ (top right plot), $30 \mathrm{~nm}$ (bottom left plot), and $300 \mathrm{~nm}$ (bottom right plot). The curves correspond to the different Geant4-DNA physics constructors (see Table I): Black circles: "option 2" constructor (default models); Green squares: "option 4" constructor (Ioannina models); Purple up-triangles: "option 6" constructor (CPA100 models); Purple down-triangles: "option 6" constructor (CPA100 models with de-activated energy-loss in elastic scattering); Blue open-circles: "Livermore" constructor; Blue open-squares: "Penelope" constructor. In both "Livermore" and "Penelope" a step-size limit of $0.5 \mathrm{~nm}$ and a secondary production-cut of $11 \mathrm{eV}$ have been used. that five electronic excitation levels and five ionization shells are considered in Geant4-DNA). Regarding "Livermore" and "Penelope" distributions, the observed sharp peaks depend on the maximum step size value and illustrate the limitation of the $\mathrm{CH}$ approach in small volumes where the modeling of energy loss fluctuations is not sufficiently accurate. Such sharp peaks are not visible for the largest scoring spheres, where the number of inelastic interactions is much larger. Clearly, with increasing sphere size, the distributions tend to become similar, independent of the physics constructor used. For the smallest sphere $(2 \mathrm{~nm})$, the $\mathrm{CH}$ simulations ("Livermore" and "Penelope" constructors) yield significantly different spectra compared to the TS simulations (Geant4-DNA). However, already from the $10 \mathrm{~nm}$ sphere, the "Livermore" spectrum resembles the Geant4-DNA spectra. For the largest sphere $(300 \mathrm{~nm})$, all physics constructors predict a sharp peak that corresponds to the entire electron energy ( $1 \mathrm{keV}$ ) being deposited within the target (independent of $\mathrm{CH}$ or TS simulation).

While these Figs. 10-13 show global agreement between spectra simulated using the three alternative sets of Geant4-DNA physics models, one should keep in mind that they exhibit different behaviors, especially at low energy. This has already been observed in this work (see Figs. 2 and 9), but also in recent comparisons. For example, ${ }^{55}$ ranges for electrons in liquid water simulated using the default models are larger than ranges simulated using the "Ioannina" models below $1 \mathrm{keV}$, due to larger electronic excitation cross sections for the "Ioannina" models. This also directly impacts dose point kernels ${ }^{55}$ of electrons in liquid water, whereby the default models appear more diffusive compared to "Ioannina" models, especially at low energy (below $500 \mathrm{eV}$ ). In addition, the "Ioannina" models have shown better agreement than the default models with Monte Carlo simulations (in the liquid phase) and measurements (in the gaseous phase) of the $\mathrm{W}$-value of water, ${ }^{54}$ a useful benchmark for the relative contribution of ionization and excitation cross sections. Very recently, we have shown that the CPA100 mod$\mathrm{els}^{56,68}$ lead also to lower ranges and less diffusive dose point kernels, compared to default Geant4-DNA models. These observations tend to favor the "Ioannina" and CPA100 models compared to default models, and still underlines the strong need for experimental data in liquid water for the full validation of Geant4-DNA physics models.

\section{CONCLUSION}

The different physics constructors available for trackstructure (TS) and condensed-history $(\mathrm{CH})$ simulation of low-energy electrons in Geant 4 have been used to calculate microdosimetry spectra and related quantities in liquid water. It was shown that the low-energy electromagnetic physics constructors ("Livermore" and "Penelope") which enable $\mathrm{CH}$ simulation in Geant4 are generally not reliable for such calculations unless careful selection of the step-size limit and secondary production-cut is made. With an appropriate choice of these two parameters, fair agreement with TS simulations using Geant4-DNA may be obtained for sphere sizes 

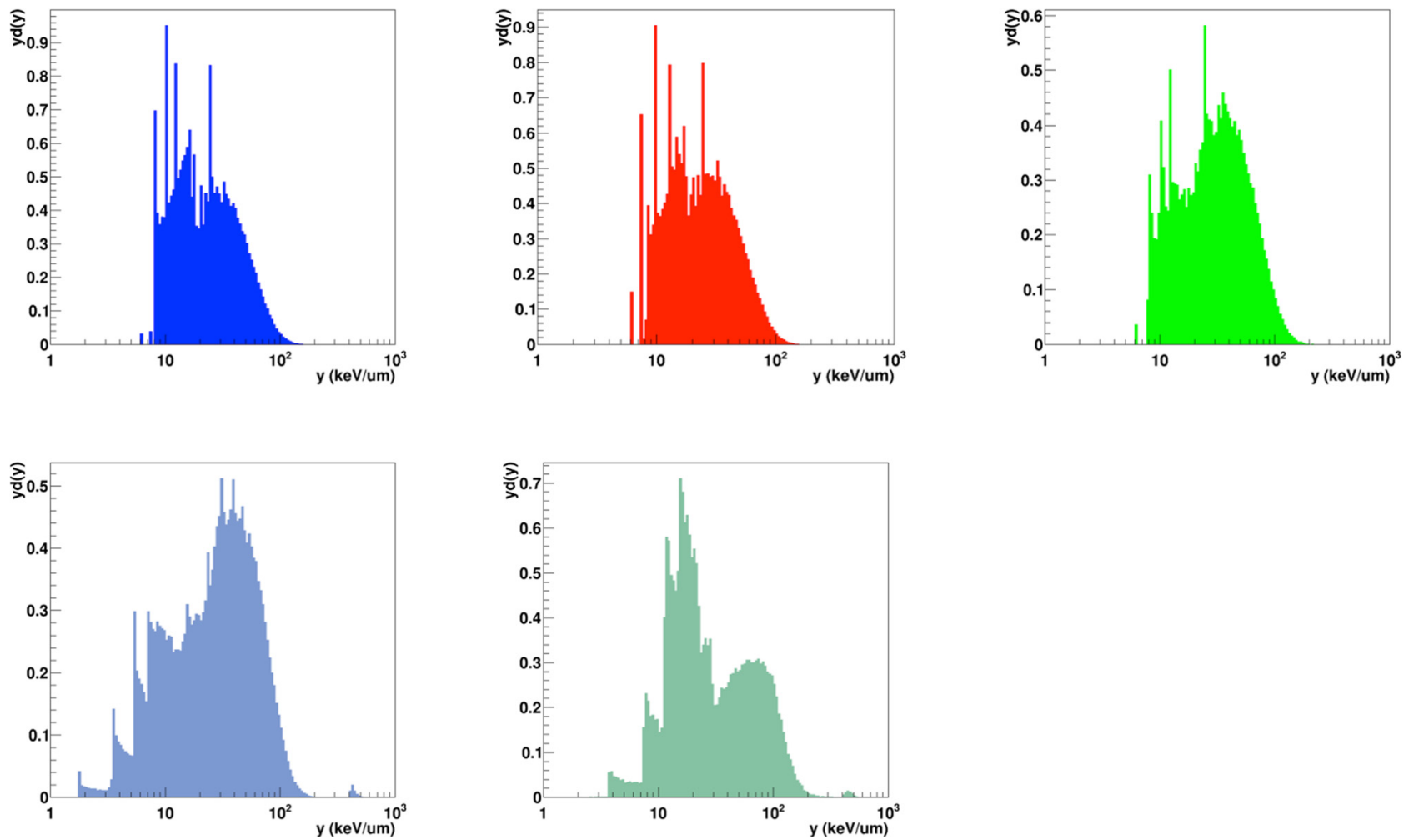

FIG. 10. Examples of $y d(y)$ versus $y$ distributions presented in logarithmic $\mathrm{x}$-scale and linear $\mathrm{y}$-scale, obtained for $1 \mathrm{keV}$ incident electrons and a $2 \mathrm{~nm}$ diameter scoring sphere. Top row: left plot corresponds to "option 2" constructor (default models), center plot to "option 4" constructor (Ioannina models) and right plot to "option 6" constructor (CPA100 models). Bottom row: left plot corresponds to "Livermore" constructor and right plot to "Penelope" constructor, using a step-size limit of $0.5 \mathrm{~nm}$ and a secondary production-cut of $11 \mathrm{eV}$. In the plots, the area delimited by two values of $y$ corresponds to the fraction of dose absorbed in that range of lineal energy.
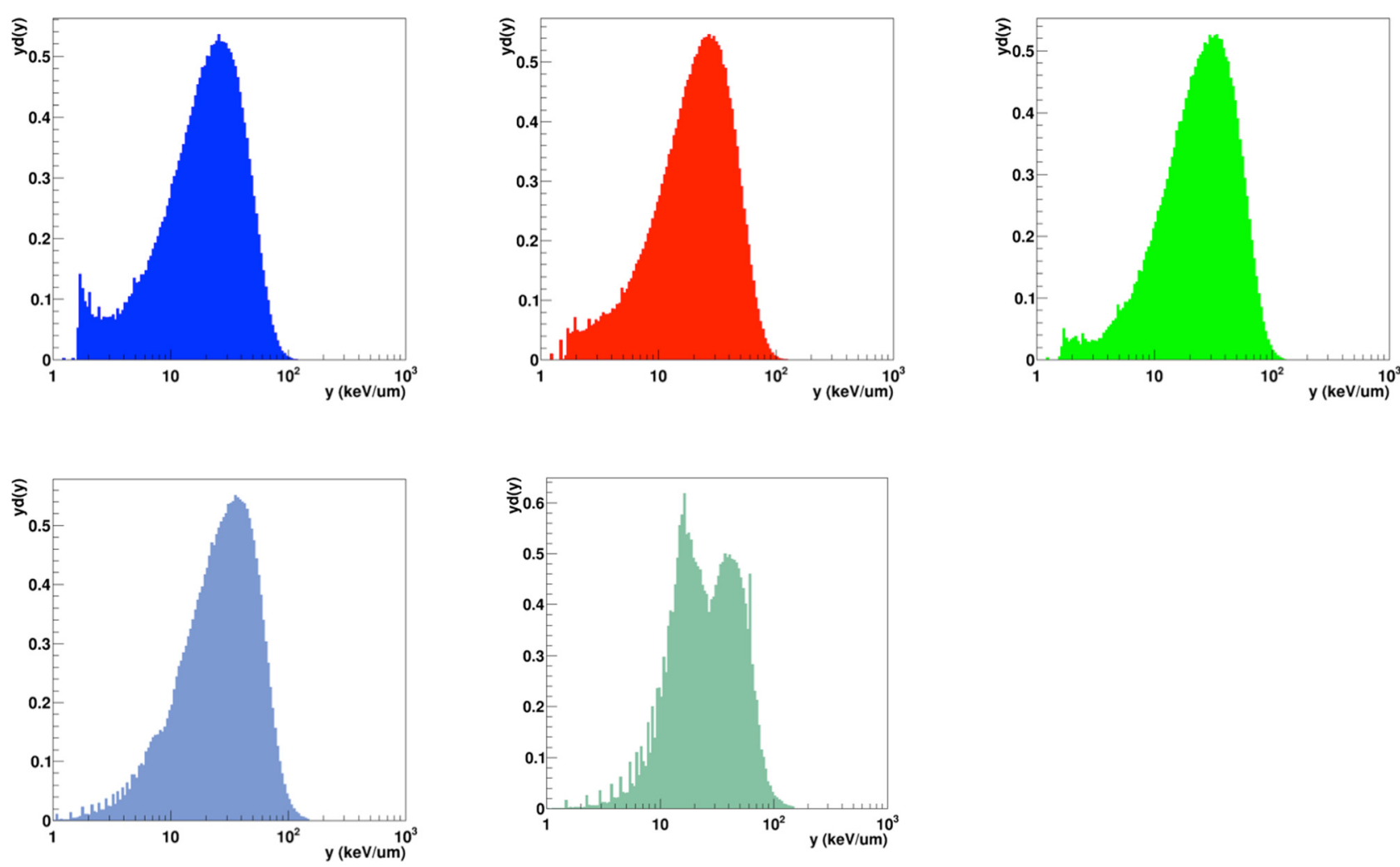

FIG. 11. Same as Fig. 10 but for a $10 \mathrm{~nm}$ diameter scoring sphere. 

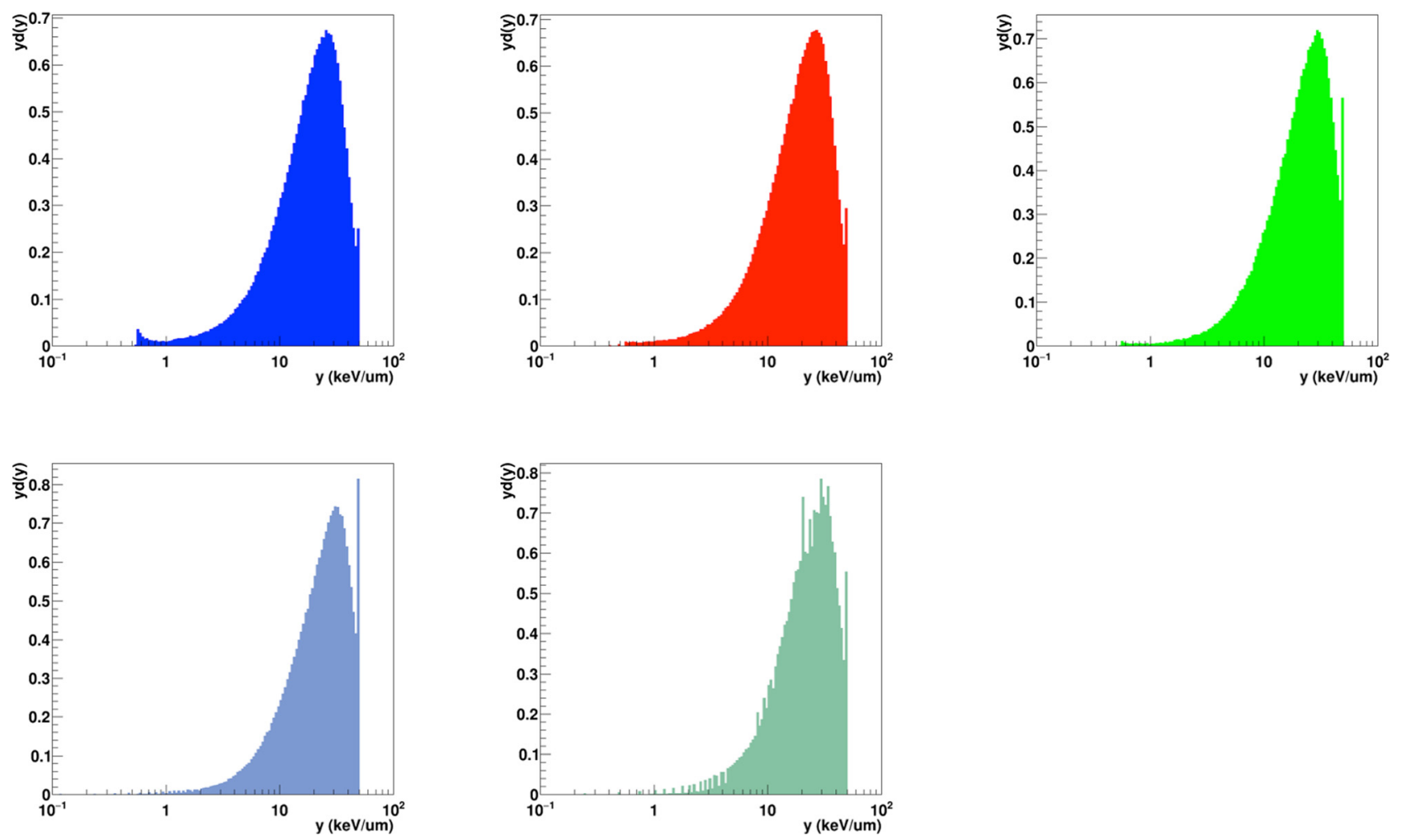

FIG. 12. Same as Fig. 11 but for a $30 \mathrm{~nm}$ diameter scoring sphere.

as small as a few tens of nanometer. In contrast, the Geant4DNA constructors (default, Ioannina, CPA100), which enable TS simulation down to about $10 \mathrm{eV}$ (or lower), are best-suited for such tasks and do not require any additional settings. Among the three Geant4-DNA constructors, the CPA100 exhibits the largest discrepancies, especially for the smaller targets examined (in the $1-10 \mathrm{~nm}$ range) owing (mainly) to the significantly different inelastic scattering
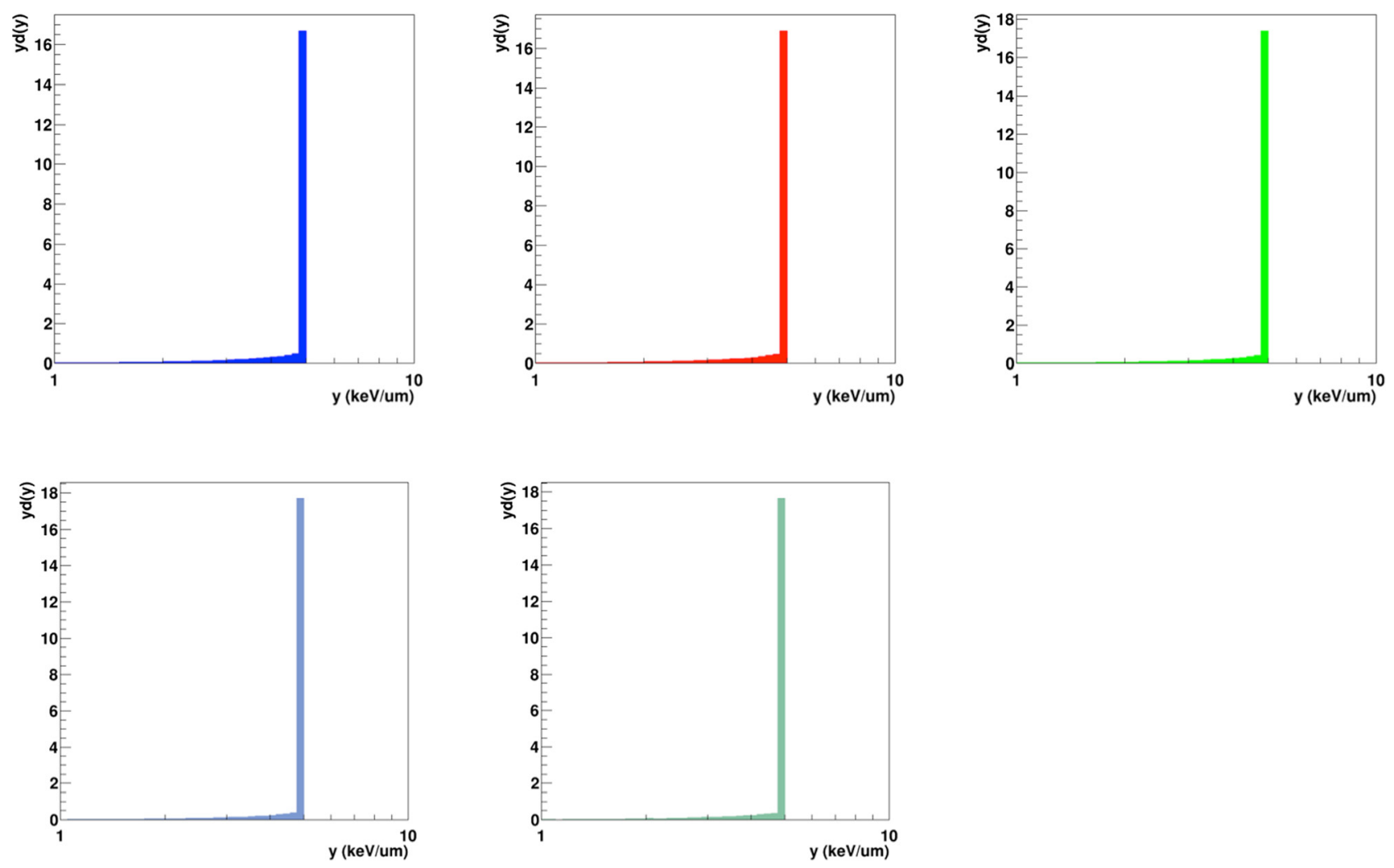

FIG. 13. Same as Fig. 12 but for a $300 \mathrm{~nm}$ diameter scoring sphere. 
model used. Finally, a new "microyz" example is discussed that could be useful for users interested in the simulation of such microdosimetry quantities using Geant4-DNA.

\section{ACKNOWLEDGMENTS}

This work was partly supported by the CNRS PICS \#7340 "Nanogold" between France and Greece.

\section{APPENDIX: DEFINITION OF MICRODOSIMETRIC QUANTITIES}

Fundamentals of microdosimetry are fully described by Kellerer in Ref. 69, which the reader is invited to consult for further detail. We present here the definition of the two stochastic quantities that we use in this work:

- the lineal energy $y$ is defined as the ratio $\varepsilon / \bar{l}$ where $\varepsilon$ is the deposited energy by a single particle track (also called "single event" ${ }^{\text {"70 }}$ ) in the probe volume (or site) of mean chord length $\bar{l}$. For a convex volume $V$ of surface $S, \bar{l}=4 \mathrm{~V} / S$. Thus, for a spherical probe, $\bar{l}=2 d / 3$ where $d$ is the sphere diameter. $y$ is usually expressed in $\mathrm{keV} / \mu \mathrm{m}$.

- the specific energy $z$ is defined as the ratio $\varepsilon / m$ where $m$ is the mass of the probe site $z$ is usually expressed in Gy.

In the case where only one incident particle is considered, lineal energy and specific energy are related quantities: for a spherical site of a unit density material $z=0.204 \frac{y}{d^{2}}$ where $d$ is expressed in micrometers, $y$ in $\mathrm{keV} / \mu \mathrm{m}$ and $z$ in Gy.

In this work, microdosimetry spectra simulated using Geant4-DNA are also quantified by means of the following non-stochastic quantities:

- the frequency-mean lineal energy $\bar{y}_{F}=\int_{0}^{\infty} y f(y) \mathrm{dy}$, where $f(y)$ is the probability density function of lineal energy $y ; \bar{y}_{F}$ is the first moment of $y$;

- the dose-mean lineal energy $\bar{y}_{D}=\frac{1}{\bar{y}_{F}} \int_{0}^{\infty} y^{2} f(y)$ dy $=\int_{0}^{\infty} y d(y) \mathrm{dy}$, where $d(y)=y f(y) / \bar{y}_{F}$ is defined as the dose-weighted probability density function of lineal energy and reflects the fact that higher lineal energies deposit a higher dose; ${ }^{71} \bar{y}_{D}$ is the second moment of $y$ divided by its first moment;

- the frequency-mean specific energy for a single event $\bar{z}_{1 F}=\int_{0}^{\infty} z f_{1}(z) d z$, where $f_{1}(z)$ is the probability density function of specific energy $z$ normalized to a single event; $\bar{z}_{1 F}$ is the first moment of $z$;

- the dose-mean specific energy for a single event $\bar{z}_{1 D}=\frac{1}{\bar{z}_{1 F}} \int_{0}^{\infty} z^{2} f_{1}(z) d z=\int_{0}^{\infty} z d_{1}(z) d z, \quad$ where $\quad d_{1}(z)=$ $z f_{1}(z) / \bar{z}_{1 F}$ is defined as the dose ("or weighted") probability density function of specific energy normalized to a single event; $\bar{z}_{1 D}$ is the second moment of $z$ divided by its first moment;

Thus, $y$ distributions apply to single events, while $z$ distributions apply to single or multi events. Note that these probability density functions all satisfy the normalization relation,

$$
\int_{0}^{\infty} f(y) d y=\int_{0}^{\infty} d(y) d y=\int_{0}^{\infty} f_{1}(z) d z=\int_{0}^{\infty} d_{1}(z) d z=1 .
$$

In the case of uniform microscopic volumes exposed to a large number of events in a uniform radiation field, the absorbed dose $D$ is obtained through the frequency-mean specific energy $\bar{z}_{F}$ for multi events

$$
D \approx \bar{z}_{F}=\int_{0}^{\infty} z f(z) d z
$$

and can also be estimated from the mean number of events $\bar{v}$

$$
D=\bar{v} \times \bar{z}_{1 F} .
$$

${ }^{1}$ H. H. Rossi and M. Zaider, Microdosimetry and Its Applications (Springer, 1996).

${ }^{2}$ J. Booz, L. Braby, J. Coyne, P. Kliauga, L. Lindborg, H. G. Menzel, and N. Parmentier, J. Int. Comm. Radiat. Units Meas. 36, 1 (1983).

${ }^{3}$ I. El Naqa, P. Pater, and J. Seuntjens, Phys. Med. Biol. 57, R75 (2012).

${ }^{4}$ T. Inaniwa, T. Furukawa, Y. Kase, N. Matsufuji, T. Toshito, Y. Matsumoto, Y. Furusawa, and K. Noda, Phys. Med. Biol. 55, 6721 (2010).

${ }^{5}$ L. Lindborg, M. Hultqvist, A. C. Tedgren, and H. Nikjoo, Phys. Med. Biol. 58, 3089 (2013)

${ }^{6}$ F. Villegas, G. Bäckström, N. Tilly, and A. Ahnesjö, Med. Phys. 43, 6322 (2016).

${ }^{7}$ L. T. Tran, L. Chartier, D. A. Prokopovich, M. I. Reinhard, M. Petasecca, S. Guatelli, M. L. Lerch, V. L. Perevertaylo, M. Zaider, and N. Matsufuji, IEEE Trans. Nucl. Sci. 62, 504 (2015).

${ }^{8}$ M. Scholz, A. M. Kellerer, W. Kraft-Weyrather, and G. Kraft, Radiat. Environ. Biophys. 36, 59 (1997).

${ }^{9}$ T. Elsässer, M. Krämer, and M. Scholz, I. J. Radiati. Oncol. Bio. Phys. 71, 866 (2008).

${ }^{10}$ Y. Kase, T. Knai, Y. Matsumoto, Y. Furusawa, H. Okamoto, T. Asaba, M. Sakama, and H. Shinoda, Rad. Res. 166, 629 (2006).

${ }^{11}$ H. Nikjoo, S. Uehara, D. Emfietzoglou, and F. A. Cucinotta, Radiat. Meas. 41, 1052 (2006).

${ }^{12}$ M. J. Berger, Methods Comput. Phys. 1, 135 (1963).

${ }^{13}$ J. F. Briesmeister, "MCNP-A general Monte Carlo Code for neutron and photon transport," Report No. LA-7396-M 3A, Los Alamos National Laboratory, Los Alamos, 1986.

${ }^{14}$ W. R. Nelson, H. Hirayama, and D. W. Rogers, "EGS4 code system," SLAC-Report-265, Stanford Linear Accelerator Center, Menlo Park, 1985.

${ }^{15}$ S. Agostinelli, J. Allison, K. Amako, J. Apostolakis, H. Araujo, P. Arce, M. Asai, D. Axen, S. Banerjee, G. Barrand, F. Behner, L. Bellagamba, J. Boudreau, L. Broglia, A. Brunengo, H. Burkhardt, S. Chauvie, J. Chuma, R. Chytracek, G. Cooperman, G. Cosmo, P. Degtyarenko, A. Dell'Acqua, G. Depaola, D. Dietrich, R. Enami, A. Feliciello, C. Ferguson, H. Fesefeldt, G. Folger, F. Foppiano, A. Forti, S. Garelli, S. Giani, R. Giannitrapani, D. Gibin, J. J. Gómez Cadenas, I. González, G. Gracia Abril, G. Greeniaus, W. Greiner, V. Grichine, A. Grossheim, S. Guatelli, P. Gumplinger, R. Hamatsu, K. Hashimoto, H. Hasui, A. Heikkinen, A. Howard, V. Ivanchenko, A. Johnson, F. W. Jones, J. Kallenbach, N. Kanaya, M. Kawabata, Y. Kawabata, M. Kawaguti, S. Kelner, P. Kent, A. Kimura, T. Kodama, R. Kokoulin, M. Kossov, H. Kurashige, E. Lamanna, T. Lampén, V. Lara, V. Lefebure, F. Lei, M. Liendl, W. Lockman, F. Longo, S. Magni, M. Maire, E. Medernach, K. Minamimoto, P. Mora de Freitas, Y. Morita, K. Murakami, M. Nagamatu, R. Nartallo, P. Nieminen, T. Nishimura, K. Ohtsubo, M. Okamura, S. O'Neale, Y. Oohata, K. Paech, J. Perl, A. Pfeiffer, M. G. Pia, F. Ranjard, A. Rybin, S. Sadilov, E. Di Salvo, G. Santin, T. Sasaki, N. Savvas, and Y. Sawada, Nucl. Instrum. Methods Phys. Res., Sect. A 506, 250 (2003).

${ }^{16}$ J. Allison, K. Amako, J. Apostolakis, H. Araujo, P. A. Dubois, M. Asai, G. Barrand, R. Capra, S. Chauvie, R. Chytracek, G. A. P. Cirrone, G. Cooperman, G. Cosmo, G. Cuttone, G. G. Daquino, M. Donszelmann, M. Dressel, G. Folger, F. Foppiano, J. Generowicz, V. Grichine, S. Guatelli, P. Gumplinger, A. Heikkinen, I. Hrivnacova, A. Howard, S. Incerti, V. Ivanchenko, T. Johnson, F. Jones, T. Koi, R. Kokoulin, M. Kossov, H. Kurashige, V. Lara, S. Larsson, F. Lei, O. Link, F. Longo, M. Maire, A. Mantero, B. Mascialino, I. McLaren, P. M. Lorenzo, K. Minamimoto, K. Murakami, P. Nieminen, L. Pandola, S. Parlati, L. Peralta, J. Perl, A. Pfeiffer, M. G. Pia, A. Ribon, P. Rodrigues, G. Russo, S. Sadilov, G. 
Santin, T. Sasaki, D. Smith, N. Starkov, S. Tanaka, E. Tcherniaev, B. Tome, A. Trindade, P. Truscott, L. Urban, M. Verderi, A. Walkden, J. P. Wellisch, D. C. Williams, D. Wright, and H. Yoshida, IEEE Trans. Nucl. Sci. 53, 270 (2006).

${ }^{17}$ J. Allison, K. Amako, J. Apostolakis, P. Arce, M. Asai, T. Aso, E. Bagli, A. Bagulya, S. Banerjee, G. Barrand, B. R. Beck, A. G. Bogdanov, D. Brandt, J. M. C. Brown, H. Burkhardt, P. Canal, D. Cano-Ott, S. Chauvie, K. Cho, G. A. P. Cirrone, G. Cooperman, M. A. Cortés-Giraldo, G. Cosmo, G. Cuttone, G. Depaola, L. Desorgher, X. Dong, A. Dotti, V. D. Elvira, G. Folger, Z. Francis, A. Galoyan, L. Garnier, M. Gayer, K. L. Genser, V. M. Grichine, S. Guatelli, P. Guèye, P. Gumplinger, A. S. Howard, I. Hřivnáčová, S. Hwang, S. Incerti, A. Ivanchenko, V. N. Ivanchenko, F. W. Jones, S. Y. Jun, P. Kaitaniemi, N. Karakatsanis, M. Karamitros, M. Kelsey, A. Kimura, T. Koi, H. Kurashige, A. Lechner, S. B. Lee, F. Longo, M. Maire, D. Mancusi, A. Mantero, E. Mendoza, B. Morgan, K. Murakami, T. Nikitina, L. Pandola, P. Paprocki, J. Perl, I. Petrović, M. G. Pia, W. Pokorski, J. M. Quesada, M. Raine, M. A. Reis, A. Ribon, A. Ristić Fira, F. Romano, G. Russo, G. Santin, T. Sasaki, D. Sawkey, J. I. Shin, I. I. Strakovsky, A. Taborda, S. Tanaka, B. Tomé, T. Toshito, H. N. Tran, P. R. Truscott, L. Urban, V. Uzhinsky, J. M. Verbeke, M. Verderi, B. L. Wendt, H. Wenzel, D. H. Wright, D. M. Wright, T. Yamashita, J. Yarba, and H. Yoshida, Nucl. Instrum. Methods Phys. Res., Sect. A 835, 186 (2016).

${ }^{18}$ A. Ferrari, P. R. Sala, A. Fasso, and J. Ranft, FLUKA: A Multi-Particle Transport Code (Program Version 2005) (CERN, Geneva, 2005).

${ }^{19}$ J. Baró, J. Sempau, J. M. Fernández-Varea, and F. Salvat, Nucl. Instrum. Methods Phys. Res., Sect. B 100, 31 (1995).

${ }^{20}$ F. Salvat and J. M. Fernández-Varea, Metrologia 46, S112 (2009).

${ }^{21}$ A. E. Nahum, Radiat. Environ. Biophys. 38, 163 (1999).

${ }^{22}$ F. Salvat, J. M. Fernández-Varea, J. Sempau, and J. Mazurier, Radiat. Environ. Biophys. 38, 15 (1999).

${ }^{23}$ R. D. Stewart, W. E. Wilson, J. C. McDonald, and D. J. Strom, Phys. Med. Biol. 47, 79 (2002)

${ }^{24}$ M. A. Bernal and J. A. Liendo, Med. Phys. 36, 620 (2009).

${ }^{25}$ J. M. Fernández-Varea, G. González-Muñoz, M. E. Galassi, K. Wiklund, B. K. Lind, A. Ahnesjö, and N. Tilly, Int. J. Radiat. Biol. 88, 66 (2012).

${ }^{26}$ M. Dingfelder, Health Phys. 103, 590 (2012).

${ }^{27}$ H. Nikjoo, D. Emfietzoglou, T. Liamsuwan, R. Taleei, D. Liljequist, and S. Uehara, Rep. Prog. Phys. 79, 116601 (2016).

${ }^{28}$ W. Friedland, M. Dingfelder, P. Kundrát, and P. Jacob, Mutation Res., Fundam. Mol. Mech. Mutagen. 711, 28 (2011).

${ }^{29}$ A. G. Georgakilas, P. O'Neill, and R. D. Stewart, Radiat. Res. 180, 100 (2013).

${ }^{30}$ R. D. Stewart, V. K. Yu, A. G. Georgakilas, C. Koumenis, J. H. Park, and D. J. Carlson, Radiat. Res. 176, 587 (2011).

${ }^{31}$ J. M. Fernández-Varea, X. Llovet, and F. Salvat, Surf. Interface Anal. 37, 824 (2005).

${ }^{32}$ M. Dingfelder, I. G. Jorjishvili, J. A. Gersh, and L. H. Toburen, Radiat. Prot. Dosim. 122, 26 (2006).

${ }^{33} \mathrm{M}$. Zaider, Physical and Chemical Mechanisms in Molecular Radiation Biology (Springer, 1991), p. 137.

${ }^{34}$ R. H. Ritchie, R. N. Hamm, J. E. Turner, H. A. Wright, and W. E. Bolch, in Physical and Chemical Mechanisms in Molecular Radiation Biology, edited by W. A. Glass and M. N. Varma (Springer US, Boston, MA, 1991), p. 99.

${ }^{35}$ M. Dingfelder, D. Hantke, M. Inokuti, and H. G. Paretzke, Radiat. Phys. Chem. 53, 1 (1999).

${ }^{36}$ D. Emfietzoglou, F. A. Cucinotta, and H. Nikjoo, Radiat. Res. 164, 202 (2005).

${ }^{37}$ M. A. Bernal, M. C. Bordage, J. M. C. Brown, M. Davídková, E. Delage, Z. El Bitar, S. A. Enger, Z. Francis, S. Guatelli, V. N. Ivanchenko, M. Karamitros, I. Kyriakou, L. Maigne, S. Meylan, K. Murakami, S. Okada, H. Payno, Y. Perrot, I. Petrovic, Q. T. Pham, A. Ristic-Fira, T. Sasaki, V. Štěpán, H. N. Tran, C. Villagrasa, and S. Incerti, Phys. Med. 31, 861 (2015).

${ }^{38}$ S. Incerti, A. Ivanchenko, M. Karamitros, A. Mantero, P. Moretto, H. N. Tran, B. Mascialino, C. Champion, V. N. Ivanchenko, M. A. Bernal, Z. Francis, C. Villagrasa, G. Baldacchino, P. Guèye, R. Capra, P. Nieminen, and C. Zacharatou, Med. Phys. 37, 4692 (2010).

${ }^{39}$ S. Incerti, G. Baldacchino, M. Bernal, R. Capra, C. Champion, Z. Francis, P. Guèye, A. Mantero, B. Mascialino, P. Moretto, P. Nieminen, C. Villagrasa, and C. Zacharatou, Int. J. Model., Simul., Sci. Comput. 01, 157 (2010).
${ }^{40}$ S. Incerti, M. Douglass, S. Penfold, S. Guatelli, and E. Bezak, Phys. Med.: Eur. J. Med. Phys. 32, 1187 (2016).

${ }^{41}$ Z. Francis, S. Incerti, R. Capra, B. Mascialino, G. Montarou, V. Stepan, and C. Villagrasa, Appl. Radiat. Isot. 69, 220 (2011).

${ }^{42}$ Z. Francis, S. Incerti, V. Ivanchenko, C. Champion, M. Karamitros, M. A. Bernal, and Z. E. Bitar, Phys. Med. Biol. 57, 209 (2012).

${ }^{43}$ L. Burigo, I. Pshenichnov, I. Mishustin, and M. Bleicher, Nucl. Instrum. Methods Phys. Res., Sect. B 320, 89 (2014).

${ }^{44}$ G. Aad, T. Abajyan, B. Abbott, J. Abdallah, S. Abdel Khalek, A. A. Abdelalim, O. Abdinov, R. Aben, B. Abi, M. Abolins, O. S. AbouZeid, H. Abramowicz, H. Abreu, B. S. Acharya, L. Adamczyk, D. L. Adams, T. N. Addy, J. Adelman, S. Adomeit, P. Adragna, T. Adye, S. Aefsky, J. A. Aguilar-Saavedra, M. Agustoni, M. Aharrouche, S. P. Ahlen, F. Ahles, A. Ahmad, M. Ahsan, G. Aielli, T. Akdogan, T. P. A. Åkesson, G. Akimoto, A. V. Akimov, M. S. Alam, M. A. Alam, J. Albert, S. Albrand, M. Aleksa, I. N. Aleksandrov, F. Alessandria, C. Alexa, G. Alexander, G. Alexandre, T. Alexopoulos, M. Alhroob, M. Aliev, G. Alimonti, J. Alison, B. M. M. Allbrooke, P. P. Allport, S. E. Allwood-Spiers, J. Almond, A. Aloisio, R. Alon, A. Alonso, F. Alonso, A. Altheimer, B. Alvarez Gonzalez, M. G. Alviggi, K. Amako, C. Amelung, V. V. Ammosov, S. P. Amor Dos Santos, A. Amorim, N. Amram, C. Anastopoulos, L. S. Ancu, N. Andari, T. Andeen, C. F. Anders, G. Anders, K. J. Anderson, A. Andreazza, V. Andrei, M. L. Andrieux, X. S. Anduaga, S. Angelidakis, P. Anger, A. Angerami, F. Anghinolfi, A. Anisenkov, N. Anjos, A. Annovi, A. Antonaki, M. Antonelli, A. Antonov, J. Antos, F. Anulli, M. Aoki, S. Aoun, L. Aperio Bella, R. Apolle, G. Arabidze, I. Aracena, Y. Arai, A. T. H. Arce, S. Arfaoui, J. F. Arguin, and E. Arik, Phys. Lett. B 716, 1 (2012).

${ }^{45}$ S. Chatrchyan, V. Khachatryan, A. M. Sirunyan, A. Tumasyan, W. Adam, E. Aguilo, T. Bergauer, M. Dragicevic, J. Erö, C. Fabjan, M. Friedl, R. Frühwirth, V. M. Ghete, J. Hammer, M. Hoch, N. Hörmann, J. Hrubec, M. Jeitler, W. Kiesenhofer, V. Knünz, M. Krammer, I. Krätschmer, D. Liko, W. Majerotto, I. Mikulec, M. Pernicka, B. Rahbaran, C. Rohringer, H. Rohringer, R. Schöfbeck, J. Strauss, F. Szoncsó, A. Taurok, W. Waltenberger, G. Walzel, E. Widl, C. E. Wulz, V. Chekhovsky, I. Emeliantchik, A. Litomin, V. Makarenko, V. Mossolov, N. Shumeiko, A. Solin, R. Stefanovitch, J. Suarez Gonzalez, A. Fedorov, M. Korzhik, O. Missevitch, R. Zuyeuski, M. Bansal, S. Bansal, W. Beaumont, T. Cornelis, E. A. De Wolf, D. Druzhkin, X. Janssen, S. Luyckx, L. Mucibello, S. Ochesanu, B. Roland, R. Rougny, M. Selvaggi, Z. Staykova, H. Van Haevermaet, P. Van Mechelen, N. Van Remortel, A. Van Spilbeeck, F. Blekman, S. Blyweert, J. D'Hondt, O. Devroede, R. Gonzalez Suarez, R. Goorens, A. Kalogeropoulos, M. Maes, A. Olbrechts, S. Tavernier, W. Van Doninck, L. Van Lancker, P. Van Mulders, G. P. Van Onsem, I. Villella, B. Clerbaux, G. De Lentdecker, V. Dero, J. P. Dewulf, A. P. R. Gay, T. Hreus, A. Léonard, P. E. Marage, A. Mohammadi, T. Reis, S. Rugovac, L. Thomas, C. Vander Velde, P. Vanlaer, J. Wang, J. Wickens, and V. Adler, Phys. Lett. B 716, 30 (2012).

${ }^{46}$ S. Incerti, Phys. Med. Biol. 59, 7565 (2014).

${ }^{47}$ D. E. Cullen, J. H. Hubbell, and L. Kissel, "EPDL97: The evaluated photon data library, 97 Version," Report No. UCRL-LR-50400-V6-R5. Lawrence Livermore National Laboratory, Livermore, 1997.

${ }^{48}$ S. T. Perkins, D. Cullen, M. Chen, J. Rathkopf, J. Scofield, and J. Hubbell, "Tables and graphs of atomic subshell and relaxation data derived from the LLNL evaluated atomic data library (EADL), $Z=1-100$," Report No. UCRL50400-V30, Lawrence Livermore National Laboratory, Livermore, 1991.

${ }^{49}$ S. Perkins, D. Cullen, and S. Seltzer, "Tables and graphs of electroninteraction cross-sections from $10 \mathrm{eV}$ to $100 \mathrm{GeV}$ derived from the LLNL evaluated electron data library (EEDL), Z = 1-100," Report No. UCRL50400-V31, Lawrence Livermore National Laboratory, Livermore, 1991.

${ }^{50}$ S. Incerti, B. Suerfu, J. Xu, V. Ivantchenko, A. Mantero, J. M. C. Brown, M. A. Bernal, Z. Francis, M. Karamitros, and H. N. Tran, Nucl. Instrum. Methods Phys. Res., Sect. B 372, 91 (2016).

${ }^{51}$ V. N. Ivanchenko, O. Kadri, M. Maire, and L. Urban, J. Phys.: Conf. Ser. 219, 032045 (2010).

${ }^{52}$ F. Salvat, J. M. Fernández-Varea, and J. Sempau, in PENELOPE-2008: A Code System for Monte Carlo Simulation of Electron and Photon Transport, Issy-les-Moulineaux, France, 2009 (OECD-NEA).

${ }^{53}$ D. Liljequist, J. Phys. D: Appl. Phys. 16, 1567 (1983).

${ }^{54}$ I. Kyriakou, S. Incerti, and Z. Francis, Med. Phys. 42, 3870 (2015).

${ }^{55}$ I. Kyriakou, M. Šefl, V. Nourry, and S. Incerti, J. Appl. Phys. 119, 194902 (2016).

${ }^{56}$ M. C. Bordage, J. Bordes, S. Edel, M. Terrissol, X. Franceries, M. Bardiès, N. Lampe, and S. Incerti, Phys. Med.: Eur. J. Med. Phys. 32, 1833 (2016). 
${ }^{57}$ D. Emfietzoglou and M. Moscovitch, Nucl. Instrum. Methods Phys. Res., Sect. B 193, 71 (2002).

${ }^{58}$ D. Emfietzoglou, K. Karava, G. Papamichael, and M. Moscovitch, Phys. Med. Biol. 48, 2355 (2003).

${ }^{59}$ D. Emfietzoglou and H. Nikjoo, Radiat. Res. 163, 98 (2005).

${ }^{60}$ M. Rudd, Y.-K. Kim, T. Märk, J. Schou, N. Stolterfoht, and L. Toburen, J. Int. Comm. Radiat. Units Meas. 55, 1 (1996).

${ }^{61}$ C. Champion, S. Incerti, H. Aouchiche, and D. Oubaziz, Radiat. Phys. Chem. 78, 745 (2009).

${ }^{62}$ S. Uehara, H. Nikjoo, and D. T. Goodhead, Phys. Med. Biol. 38, 1841 (1993).

${ }^{63}$ Y.-K. Kim and M. E. Rudd, Phys. Rev. A 50, 3954 (1994).

${ }^{64}$ X. Wang, MSc thesis, Texas A\&M University, 2006.

${ }^{65}$ J. Dicello and F. Cucinotta, see https://three.jsc.nasa.gov/articles/ MICRODOSIMETRY.pdf for "Interpreting microdosimetric spectra" (last accessed May 18, 2017).
${ }^{66}$ R. Brun and F. Rademakers, Nucl. Instrum. Methods Phys. Res., Sect. A 389, 81 (1997).

${ }^{67}$ G. Baiocco, S. Barbieri, G. Babini, J. Morini, D. Alloni, W. Friedland, P. Kundrát, E. Schmitt, M. Puchalska, L. Sihver, and A. Ottolenghi, Sci. Rep. 6, 34033 (2016)

${ }^{68}$ J. Bordes, S. Incerti, N. Lampe, M. Bardiès, and M.-C. Bordage, Nucl. Instrum. Methods Phys. Res., Sect. B 398, 13 (2017).

${ }^{69}$ A. M. Kellerer, The Dosimetry of Ionizing Radiation (Academic Press, 1985), p. 77

${ }^{70} \mathrm{P}$. Olko, Habilitation thesis, Henryk Niewodniczański Institute of Nuclear Physics, 2002.

${ }^{71}$ A. B. Rosenfeld, P. D. Bradley, I. Cornelius, G. I. Kaplan, B. J. Allen, J. B. Flanz, M. Goitein, A. V. Meerbeeck, J. Schubert, J. Bailey, Y. Takada, A. Maruhashi, and Y. Hayakawa, IEEE Trans. Nucl. Sci. 47, $1386(2000)$. 